Citation: E. Dai Prà, N. Gabellieri (2020) Imago proelii. La cartografia storica della Prima Guerra Mondiale dal Museo Storico della Terza Armata: spigolature per una analisi tipologica e semiologica. Bollettino della Società Geografica Italiana serie 14, 3(1): 59-79. doi: 10.36253/bsgi.v3i1.445

Copyright: (c) 2020 E. Dai Prà, N. Gabellieri. This is an open access, peer-reviewed article published by Firenze University Press (http://www. fupress.com/bsgi) and distributed under the terms of the Creative Commons Attribution License, which permits unrestricted use, distribution, and reproduction in any medium, provided the original author and source are credited.

Data Availability Statement: All relevant data are within the paper and its Supporting Information files.

Competing Interests: The Author(s) declare(s) no conflict of interest.

For Italian evaluation purposes: E. Dai Prà takes responsability for sections 1, 5 and 6, and N. Gabellieri for sections 2,3 and 4 .

\section{Imago proelii. La cartografia storica della Prima Guerra Mondiale dal Museo Storico della Terza Armata: spigolature per una analisi tipologica e semiologica}

\author{
Imago proelii. The historical maps of the First World War in the \\ Terza Armata Historical Museum: clues for a typological and \\ semiological analysis
}

\author{
Elena Dai Prà, Nicola Gabellieri \\ Dipartimento di Lettere e Filosofia, Università di Trento, Italia \\ E-mail: elena.daipra@unitn.it; nicola.gabellieri@unitn.it
}

\begin{abstract}
International historiography has extensively assessed the innovations in war practice experienced during the First World War. However, the consequences of trench warfare in military cartographic production have not been fully investigated yet. Following this line, the essay presents the cartographic corpus of the historical archive of the Terza Armata (Third Army); the Terza Armata had a fundamental role in the conflict, settling on the Piave river after Caporetto and leading the final advance towards Istria. The analysis of the collection, which is still unpublished, can open a new front of research aimed at developing categories of semiological analysis and typological classification of military maps as sources produced in fieri, i.e. manuscript IGM maps that were constantly updated during war operations. First, the cartographic corpus is presented; second, a first typological classification and a semiological decoding of maps are proposed, based on the interpretation of the symbols used, of the legend and of the methods of representation. In particular, the contribution focuses on tactical maps, i.e. cartographies that were continuously updated in proelio to document the evolution of war events on the front line.
\end{abstract}

Keywords: Historical cartography, First World War, tactical military cartography, military archives, maps typological classification.

\footnotetext{
Riassunto. La storiografia internazionale ha ampiamente documentato le innovazioni nella prassi bellica sperimentate durante il primo conflitto mondiale. Non ancora ben approfondite sono invece le conseguenze delle necessità della guerra di trincea nella produzione cartografica militare. A questo proposito, il contributo presenta il patrimonio cartografico attualmente custodito presso l'Archivio storico della Terza Armata, che assunse un ruolo fondamentale nel conflitto, attestandosi sul Piave dopo Caporetto e guidando l'avanzata finale verso l'Istria. L'esegesi della raccolta, ancora inedita, vuole aprire un nuovo fronte di ricerca diretto all'elaborazione di categorie di analisi semiologica e classificazione tipologica delle carte militari come fonti prodotte in fieri, ossia mappe di impianto IGM costantemente aggiornate in forma manoscritta durante le
} 
operazioni. In primo luogo si presenta, quindi, il corpus cartografico; in secondo luogo, si propone una prima classificazione tipologica e un tentativo di decodifica semiologica dei documenti, basato sull'interpretazione della simbologia utilizzata, della legenda e dei metodi di rappresentazione. In particolare, il contributo si sofferma sulle carte tattiche, ovvero quelle continuamente aggiornate in proelio per documentare l'evolversi delle vicende belliche in prima linea.

Parole chiave: Cartografia storica, Prima Guerra Mondiale, cartografia militare tattica, archivi militari, classificazione tipologica della cartografia.

\section{Geografia, cartografia, guerra: un rapporto atavico in evoluzione}

Fin dagli albori della loro storia, ars bellica e ars geographica hanno sempre mantenuto uno stretto legame: "La géographie, ça sert, d'abord, à faire la guerre" (Lacoste 1976). Se per lungo tempo la conoscenza territoriale è stata direttamente soggetta alle attività politiche e militari, a partire dall'età contemporanea l'evoluzione del concetto e della pratica di guerra e la progressiva professionalizzazione degli studi geografici e della produzione cartografica hanno portato a una rimodulazione - ma non certo ad una attenuazione - di questo legame (Francis 2014). In questo percorso, la Prima Guerra Mondiale ha rappresentato uno spartiacque significativo, vista la mobilitazione senza precedenti che ha coinvolto la scienza e la tecnologia nel conflitto, e le innovazioni sviluppate durante il suo decorso, tanto da essere stata definita come "indeed a terribile mother of invention" (Demhardt 2018, 241; cfr. Collier 2015a e Fox 2018); ben note sono le novità tattiche introdotte, come le trincee, il combattimento aereo, i primi mezzi corazzati, i gas tossici. L'approssimarsi della ricorrenza del centenario della Grande Guerra ha costituito, a livello internazionale, l'occasione per aprire nuove domande anche sul ruolo giocato dai saperi e dalle competenze geografiche prima, durante e dopo il confronto bellico. I geografi parteciparono attivamente ai vari dibattiti interventisti, nazionalisti o pacifisti pre-conflitto e alla preparazione degli studi conoscitivi pre-bellici (Proto 2014; Laureti 2018); prestarono servizio al fronte come semplici soldati o esercitando le proprie conoscenze nei rilevamenti, nella fotointepretazione e nelle esplorazioni (Rombai 2016; Reali, Ricci 2018; Gabellieri 2019); nelle retrovie continuarono a produrre cartografie, o a raccogliere informazioni sul nemico servendo nei sistemi di intelligence (Heffernan 1996; Maddrell 2008); ebbero un ruolo fondamentale nella propaganda, anche attraverso la produzione di car- te specificatamente pensate all'uopo (Boria 2012); contribuirono, dopo la cessazione delle ostilità, alla delineazione dei nuovi confini e alla gestione dei territori conquistati (Ferrandi 1986).

Rispetto alla storia della cartografia militare, il problema della Grande Guerra come spartiacque è stato sollevato nel dibattito geografico-storico internazionale nel 2014 dal 5th International Symposium della International Cartographic Association (Liebenberg et al. 2016), che ha inaugurato un percorso di ricerca culminato in un numero speciale dell'International Journal of Cartography del 2018 (Demhardt 2018) con saggi dedicati alle cartografie dell'esercito britannico, statunitense, russo, tedesco e austroungarico, oltre ad un approfondimento sulla fotogrammetria. Stupisce, in questo come in altre rassegne collettanee o saggi di sintesi internazionali sul tema (Chasseaud 2013; Collier 2015b; Schneider 2017), la quasi totale assenza di contributi sul caso studio italiano rispetto alla fortuna che hanno avuto le analisi di produzioni cartografiche degli stati alleati o ostili. I geografi italiani sono stati però promotori nel triennio 2015-2018 di numerosi convegni accademici e iniziative editoriali, coordinate perlopiù dal Centro Italiano per gli Studi Storico-Geografici (CISGE), che hanno permesso di colmare numerose lacune nella storia della geografia e della cartografia di quel periodo ${ }^{1}$.

Tale ricco e fruttuoso percorso di studi ha permesso di esplicitare come la Prima Guerra Mondiale sia stata combattuta innanzitutto sui tavoli dei cartografi. Corografie e topografie costituirono strumenti fondamentali non solo per agevolare la comprensione del terreno di battaglia e per la pianificazione di strategie a piccola scala, ma anche medium vitali nella dimensione tattica quotidiana. Le carte disponibili all'inizio del conflitto, perlopiù legate alle necessità delle campagne di matrice ottocentesca, risultarono ampiamente insufficienti dal punto di vista informativo per operazioni quotidiane sul campo. La scienza cartografica dovette confrontarsi con le nuove esigenze dettate dalla guerra di posizione; piegarsi a necessità strategiche e tattiche come la preparazione delle opere difensive, la pianificazione delle offensive, la direzione del tiro delle artiglierie, il reperimento e la mobilitazione delle risorse; legarsi alle nuove tecniche di rilevamento aereo e alla produzione industriale. La capacità di ogni Stato Maggiore dei vari eserciti di sviluppare prassi aerofotogrammetriche e di produzione cartografica costituì uno degli elementi cruciali di

${ }^{1}$ Dai Prà 2018; Masetti 2018; Chirico, Conti 2018; Bondesan, Scroccaro 2016; per quanto riguarda un lavoro di sintesi sulla storia della cartografia italiana, la dettagliata rassegna di Andrea Cantile (2013) si interrompe agli albori del conflitto mondiale. 
successo bellico, al pari degli altri avanzamenti tecnicoscientifici, in un graduale processo di professionalizzazione cartografica.

L'esperienza della guerra di posizione ha cambiato fondamentalmente le domande poste al sapere geografico, e soprattutto alla produzione cartografica, tanto che, al termine del conflitto, i geografi militari e gli Stati Maggiori di tutti gli eserciti belligeranti hanno concordato che questa esperienza aveva prodotto un completo "geographical turn of mind" (The map sense 1915, 9). Per dipanare il filo dell'intricata matassa rappresentata dal tema del ruolo e dello sviluppo delle cartografie storiche durante la Grande Guerra, è sembrato interessante approcciare criticamente un corpus cartografico unitario, custodito presso l'archivio del Museo Storico della $3^{\text {a }}$ Armata. Il materiale reperito, analizzato nella sua totalità, ha permesso di delineare un primo quadro classificatorio delle tipologie di carte e aerofotogrammetrie utilizzate e di tracciare dei primi spunti per una analisi tipologica e semiologica delle simbologie registrate su questi documenti. L'obiettivo è articolare problemi, riflessioni e spunti che possano auspicabilmente aprire un lungo percorso di ricerca sulla produzione di cartografia in tempo di guerra e su come il primo conflitto "industriale" abbia comportato lo sviluppo di nuovi habitus e prassi per vedere e rappresentare cartograficamente lo spazio bellico topografico.

\section{Fonti cartografiche militari e istituti di conservazione}

Nel nostro paese, la conservazione dei materiali documentari militari rappresenta un nodo parzialmente insoluto, nonostante gli sforzi compiuti in questa direzione dall'Ufficio Storico dell'Esercito e dall'Istituto Geografico Militare. La gran parte dei documenti storici prodotti dall'Esercito Italiano rimane ancora oggi res incognita per molteplici motivi, legati alla natura stessa del peculiare contesto archivistico e alla sua storia. In primo luogo, la documentazione prodotta in tempo di guerra è stata oggetto di alterne vicende come conseguenza del suo valore strategico, che ne hanno condizionato continui traslochi, dispersioni o addirittura distruzioni da parte dei suoi utenti a seconda delle vicende belliche e per i motivi più disparati. In secondo luogo, le carte della Prima e della Seconda Guerra Mondiale sono considerate documenti ancora correnti e, come tali, non sottoposti ai vincoli e ai versamenti agli archivi di Stato dettati dal Ministero dei Beni Culturali. Gran parte di tali fonti rimangono quindi dispersi in numerosi sedi; questo peculiare status conservativo ha comportato anche una certa parzialità nella selezione dei documenti da trasmettere all'Ufficio Storico dello Stato Maggiore e la perdita di alcuni di essi a causa di contesti di custodia non adatti, anche a causa dell'annoso problema, comune a tutti gli archivi italiani, della mancanza di spazio (Trani 2013). La parziale disponibilità e il difficile accesso agli studiosi sono, a giudizio dello storico Nicola Labanca (2014), tra le cause che hanno ostacolato lo sviluppo di ricerche storiche italiane nel campo della storia militare contemporanea, anche rispetto ad altre esperienze come quella degli storici francesi, e possono in una certa misura concorrere a spiegare la mancanza nel nostro paese di una solida tradizione di studi sulla cartografia storica militare (Trani 2006).

Eppure, negli ultimi anni si è sviluppata anche in ambito militare una più illuminata consapevolezza della natura dei documenti storici come fonti, che ha portato all'elaborazione di più affinati strumenti di ricerca, di metodologie archivistiche e di regolamenti interni sulla loro consultazione e sul loro versamento (Carucci 2013), estesi anche a istituti di conservazione non strettamente militari (Fontana, Pisetti 2019). Di fatto, queste lodevoli iniziative si rendono di difficile attuazione a causa della complessa composizione dell'universo degli archivi militari; sotto questa definizione ricade infatti un complesso eterogeneo di corpi dell'Esercito ed enti di musealizzazione, come le sedi dei Comandi, dei Distretti e i musei militari a gestione pubblica o privata. Tra di essi si può considerare il Museo Storico della $3^{\text {a }}$ Armata, sito a Padova.

La $3^{\text {a }}$ Armata ebbe un ruolo cruciale nel conflitto, in quanto, ritirata "invitta" dopo Caporetto, agì da fronte di contenimento sul Piave, partecipò alla battaglia di Vittorio Veneto e guidò l'avanzata finale fino all'Istria (Jung 2000; Gibelli 2007). Il Museo intitolato, istituito nel 1956 per iniziativa del Generale Alberto Aliberti e dipendente dal Comando Forze di Difesa Interregionale Nord, rappresenta l'unico ente espositivo dell'Esercito Italiano dedicato ad un'Armata specifica. Oltre ai cimeli esposti nelle sale su due piani di Palazzo Camerini, il Museo custodisce numerose fonti - solo parzialmente inventariate - prodotte durante la Prima Guerra Mondiale dal Comando Centrale o dagli uffici e corpi subalterni della $3^{\text {a }}$ Armata, tra cui circa 13.000 buste di documenti testuali, 4.000 volumi, 5.000 foto tra stereoscopie e cartacee e 1.000 cartografie, con particolare riferimento all'area del Carso e del Basso Piave. Questo corpus documentale ha una composizione eterogenea che comprende album fotografici, rapporti, giornali di trincea, manoscritti di ufficiali e subalterni, bollettini di guerra, comunicati al Comando, telegrammi, colombigrammi, corrispondenza varia e aereofotogrammetrie. Nonostan- 
te tale eterogeneità, questo fondo costituisce un caso di studio paradigmatico e dalle grandi potenzialità, data la sua natura unitaria dettata dal soggetto produttore e il buono stato di conservazione, frutto dell'encomiabile impegno nella sua custodia da parte della Direzione del Museo e della Associazione Amici del Museo Storico della $3^{\text {a }}$ Armata.

\section{La cartografia della Prima Guerra Mondiale}

All'inizio del XX secolo in ogni Stato europeo la pianificazione bellica era fortemente legata alla conoscenza e alla mappatura del territorio. Le cartografie militari potevano essere suddivise in due macrotipologie: quelle strategiche, diffuse sin dalla prima età moderna, che riproducevano caratteristiche topografiche come corsi d'acqua, viabilità, insediamenti e copertura boschiva, utilizzate per pianificare le operazioni belliche ad ampio raggio; e le mappe dei campi di battaglia, redatte sia per illustrare assetti bellici pregressi, sia per pianificare operazioni militari specifiche (Liebenberg et al. 2016). A livello internazionale, si assisteva ad una forte eterogeneità sia in termini di conoscenze cartografiche, sia per quanto riguardava le caratteristiche dei documenti come la scala, il sistema di coordinate e di proiezione e la simbologia (Schneider 2017; Collier 2015a; Chasseaud 2018).

L'Esercito Italiano poteva contare sui fogli e sui quadranti della Grande Carta Topografica del Regno d'Italia, prodotti dall'IGM alle scale di 1:100.000 e 1:50.000, con alcune tavolette di maggior dettaglio alla scala 1:25.000 per aree di particolare interesse strategico (Cantile 2019; Guarducci 2018). Inizialmente i comandi vennero dotati solo della carta a scala 1:100.000 (Ministero della Guerra, 1912, p. 50); a seguito dell'andamento assunto dal conflitto e delle innovazioni belliche, questo tipo di dotazione cartografica si rivelò presto insufficiente a rispondere alle necessità delle truppe al fronte, analogamente a quanto accadde per gli eserciti alleati o ostili (Bondesan 2016; Guarducci 2018). Anche se ogni Stato Maggiore aveva già a disposizione dei corpi deputati a rilevare e cartografare il terreno, tutti i belligeranti dovettero rapidamente organizzare centri in prima linea con il compito di reperire informazioni e realizzare e stampare le carte necessarie con gli aggiornamenti del caso. In Italia ogni Armata distribuita sul fronte austriaco si dotò nel corso dei primi due anni di guerra di un Ufficio Informazioni Truppe Operanti (UITO), diviso in Ufficio Situazione Truppe Nemiche, Ufficio Interpretazione Foto Aeree e Ufficio Cartografia, che fornivano informazioni al Comando di Corpo D'Armata e all'Uf- ficio Situazione Artiglieria. Vari distaccamenti dello UITO presso ogni reggimento e brigata sul fronte, i Centri Raccolta Informazioni Truppe Operanti (CRITO), trasmettevano a loro volta dati e aggiornamenti raccolti sul fronte all'ufficio centrale (Gabellieri, Dai Prà 2020).

La diffusione di inedite infrastrutture e dotazioni belliche, come le trincee o le teleferiche, comportò lo sviluppo di una nuova simbologia cartografica per la loro rappresentazione. Inizialmente questi nuovi simboli vennero sviluppati individualmente da ciascun esercito, per poi essere codificati e normati in nuovi standard cartografici alla fine del conflitto (Schneider 2017). Inoltre, il rapido mutare delle posizioni, insieme alla precisione richiesta per il tiro delle artiglierie - che, come è stato sottolineato, erano posizionate in seconda linea e non più fronteggianti le truppe, e quindi necessitavano di un accurato calcolo delle traiettorie (Collier, 2015b; Reali, Ricci 2018) - resero necessaria l'adozione di accurate carte a grande scala aggiornate quotidianamente e che rappresentassero quanto più dettagliatamente possibile la posizione del nemico con le relative datazioni. Per sviluppare tale dotazione, una possibilità era quella di utilizzare ed adattare le carte redatte dal nemico, ottenute grazie ad azioni di intelligence o conquista di trincee, oppure di reperire dati attraverso gli interrogatori dei prigionieri (Ministero della Guerra, 1912, 53). La vera innovazione della Grande Guerra in campo cartografico fu però l'utilizzo estensivo delle rilevazioni aeree, grazie all'affinamento di nuove apparecchiature fotografiche da montare sugli apparecchi e allo sviluppo di nuove tecniche di fotogrammetria, rettificazione e interpretazione delle foto aeree zenitali o oblique; innovazioni che contribuirono alla nascita della scienza fotogrammetrica nel periodo del dopoguerra (Espenhorst 2016; Collier 2018). Dalle foto aeree e dalle telefotografie era possibile trarre elementi informativi di interesse tattico, quali movimenti di truppe, accantonamenti, posizioni delle batterie o delle singole bocche da fuoco delle artiglierie nemiche e finanche di aperture sui fianchi delle montagne, indicative della presenza di gallerie e di postazioni armate, mentre sul piano strategico le stesse foto mostravano, con giorni e settimane di anticipo, l'approntamento di grandi manovre nelle retrovie, attraverso l'osservazione sistematica dei movimenti ferroviari e stradali di uomini e mezzi (Carbone, Ciaschi 2018; Collier 2018; Allegri 2019). A questo proposito, l'Esercito Italiano poteva vantare una tradizione più consolidata rispetto ai paesi alleati o ostili, grazie alle campagne di rilevamento delle aree di frontiera compiute dall'IGM fin dalla fine dell'Ottocento con le macchine fotografiche RosterMariani, alle sperimentazioni di applicazione di apparati telefotografici applicati a dirigibili e aerei della Sezione 
Fotografica del Genio Militare nata nel 1896 e alle esperienze sull'uso degli aerei in attività belliche maturate nella Guerra di Libia (Bergaglio 2016; Cantile 2019).

Le informazioni tattiche e strategiche desunte dai rapporti delle truppe, o ottenute grazie alla fotointerpretazione e ai rilevamenti da terra, venivano riportate in forma manoscritta su basi composte da tavole IGM, usando matite o pastelli. Ogni carta topografica era poi dotata di una apposita legenda, necessaria in un momento in cui la tassonomia simbolica non era ancora adeguatamente codificata, e di una data (e a volte anche dell'ora) che ne dimostra la natura transitoria; questo tipo di documenti sono stati definiti "carte tattiche", ovvero prodotte in proelio per rappresentare lo stato coevo del dispiegamento delle forze (Traversi 1968; cfr. Espenhorst 2018 e Chasseaud 2018). Se, alla vigilia del conflitto, lo Stato Maggiore poteva contemplare la possibilità che "in ogni comando di grande unità può giovare che durante il combattimento un ufficiale [...] segni su una carta le posizioni che dal complesso delle informazioni risultano occupate dalle proprie truppe e dalle truppe nemiche nei momenti più salienti dell'azione" (Ministero della Guerra 1912, 53), questa pratica divenne rapidamente la prassi. Per rispondere a questa esigenza, l'Istituto Geografico Militare, attraverso un notevole impegno di uomini e di risorse, stampò e distribuì in prima linea venti milioni di carte, perfezionando anche riduzioni delle tavolette al 10.000 e al 5.000 attraverso ingrandimenti fotomeccanici ${ }^{2}$.

\section{Il materiale cartografico del Museo della $3^{\text {a }}$ Armata: proposte di esegesi e classificazione}

Il fondo cartografico custodito negli archivi del Museo della $3^{\text {a }}$ Armata costituisce un corpus documentario complesso. Le carte ivi contenute sono molto eterogenee, in quanto elaborate con procedimenti diversi, per rispondere a tutte le differenti necessità dei vari corpi durante le operazioni belliche. Inoltre, molte di esse, prodotte per rappresentare la situazione, in continuo mutamento del campo di battaglia, erano continuamente sostituite e sono state distrutte con il protrarsi del conflitto. Per questo motivo, la maggior parte di quelle che ci sono pervenute risalgono agli ultimi due anni di guerra. Questi "vuoti documentali" rendono difficile ricostruire l'evoluzione del loro stile e del loro linguaggio. Per una loro piena comprensione, si aprono una serie di "sfide"

\footnotetext{
${ }^{2}$ Cantile 2019, 37. Tale numero è comunque inferiore ai 32 milioni stimati per il Regno Unito, ai 40 milioni della Francia e alle 700 milioni di carte della Germania (Espenhorst 2018, 309).
}

euristiche: la necessità di comprendere le loro tassonomie e legende; di interpretare semiologicamente i simboli utilizzati; di contestualizzare ogni carta, ovvero di identificarne la data, le modalità di produzione, lo scopo, gli usi, gli autori; la classificazione in diverse tipologie.

Per quanto riguarda questo ultimo tema, la documentazione è divisibile in due diversi macro-gruppi: cartografie e aerofotogrammetrie (Tab. 1).

\subsection{Le fotografie aeree e topografiche}

Dopo i primi mesi di guerra la fotografia aerea passò da essere procedimento sperimentale a pratica consolidata ampiamente utilizzata dagli apparati aerei delle armate e dai sistemi informativi di spionaggio. Foto aeree zenitali o oblique, scattate durante le ricognizioni, erano lette e interpretate nelle retrovie e utilizzate per aggiornare continuamente le carte tattiche e strategiche, sia per lo spionaggio delle posizioni nemiche, sia per $\mathrm{i}$ rilevamenti e il monitoraggio delle proprie forze. Durante la guerra, l'aviazione non costituiva un corpo autonomo dall'esercito; la $3^{\text {a }}$ Armata aveva in gestione una propria forza aerea, equiparata ai reggimenti di cavalleria. Dai quattro campi di aviazione di Quinto, Marcon, Ca' Tessera e Malcontenta, situati vicino a Venezia e Treviso, decollavano gli aerei in dotazione all'armata, con compiti di ricognizione, mitragliamento e perfino sperimentazione di bombardamenti tattici. La fig. 1 mostra l'assemblaggio delle strisciate di volo realizzate dal capitano Natale Palli su Trieste nel luglio 1918. Le foto, scattate in zenitale a una quota di $1.000 \mathrm{~m}$., sono state assemblate su carta secondo il piano di volo. La bassa quota indica, oltre alla perizia del pilota, il forte interesse per i dettagli tattici rispetto al quadro complessivo della città. Immediatamente identificabili sono le navi da guerra alla fonda o di pattuglia, insieme alla articolazione delle banchine per le riparazioni e dei cantieri navali. Natale Palli fu, insieme a Gabriele D'Annunzio, anche il protagonista della più importante operazione propagandistica aerea della Grande Guerra, ovvero il volo su Vienna compiuto nell'agosto del 1918 (Gentilli, Varriale 1999). Il Museo custodisce anche le foto scattate in questa occasione, che riproducono il Castello di Schönbrunn (Fig. 2). In questo caso, le immagini (scattate da quota 800 m.) sono state montate su carta a ricostruire una panoramica del castello, ma con un chiaro intento propagandistico piuttosto che bellico, ovvero a dimostrazione del compiuto raid e della superiorità aerea italiana su quella austroungarica.

Una seconda categoria di foto sono le immagini scattate dal terreno in prima linea, dalle trincee o dai 


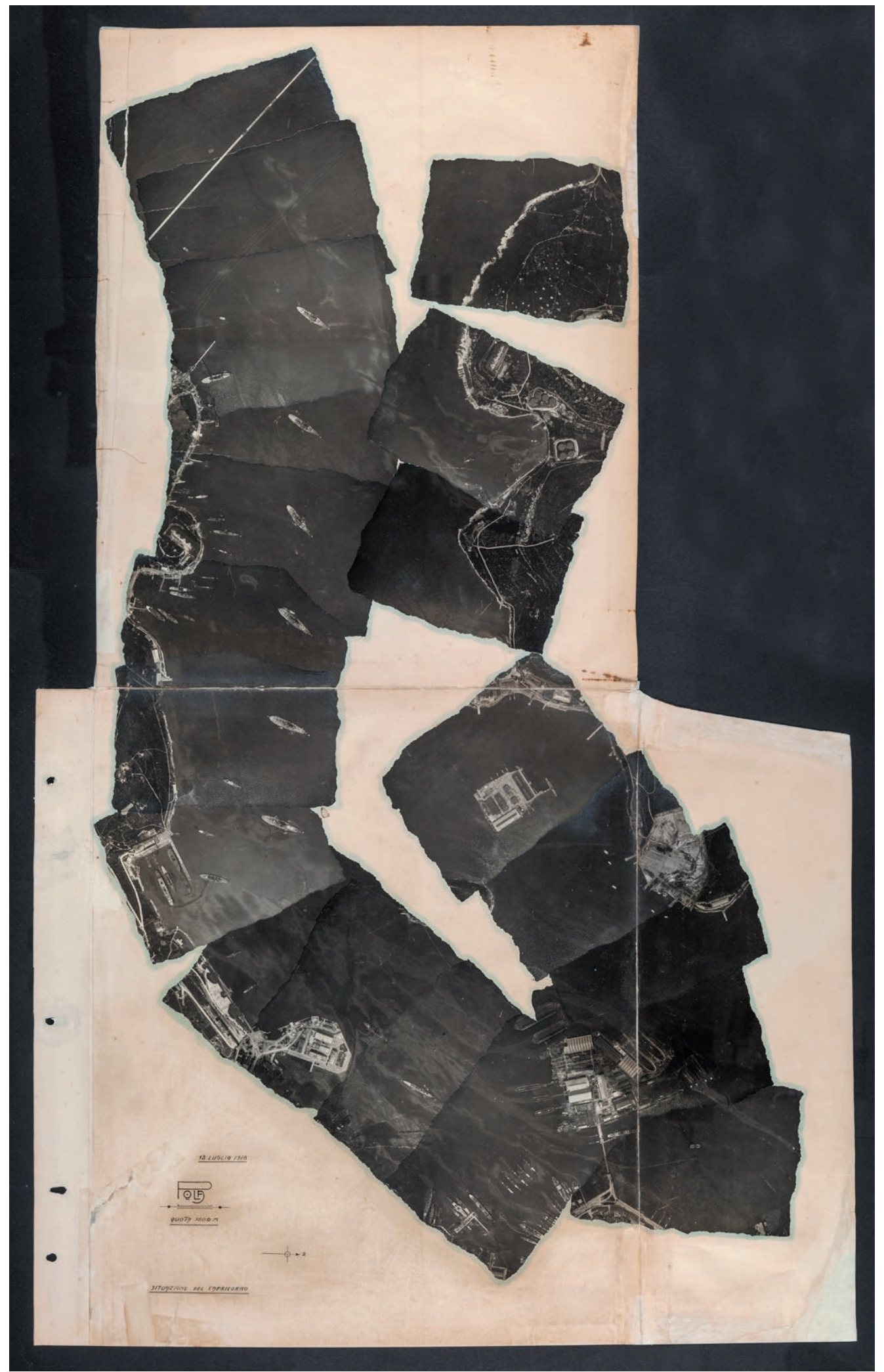

Figura 1. Foto zenitali del porto di Trieste, 13 luglio 1918, autore Cap. Natale Palli. Fonte: Archivio del Museo della $3^{\mathrm{a}}$ Armata, Padova, Fondo cartografico, Foto aeree Cap. Palli, 28. 
rilievi, per ottenere informazioni sulle caratteristiche della "terra di nessuno", delle trincee e delle ridotte e delle posizioni di artiglieria, punti deboli e forti dello schieramento, preparatorie alle offensive. Molte di esse sono riproduzioni panoramiche, di ampie dimensioni; sulla foto erano poi trascritti i toponimi - sia civili sia militari - dei punti notevoli, come le città e le frazioni, la morfologia o gli elementi strategici. Ad esempio, la panoramica della fig. 3 porta come titolo la denominazione "Panorama dell'Hermada da q. 93", che identifica

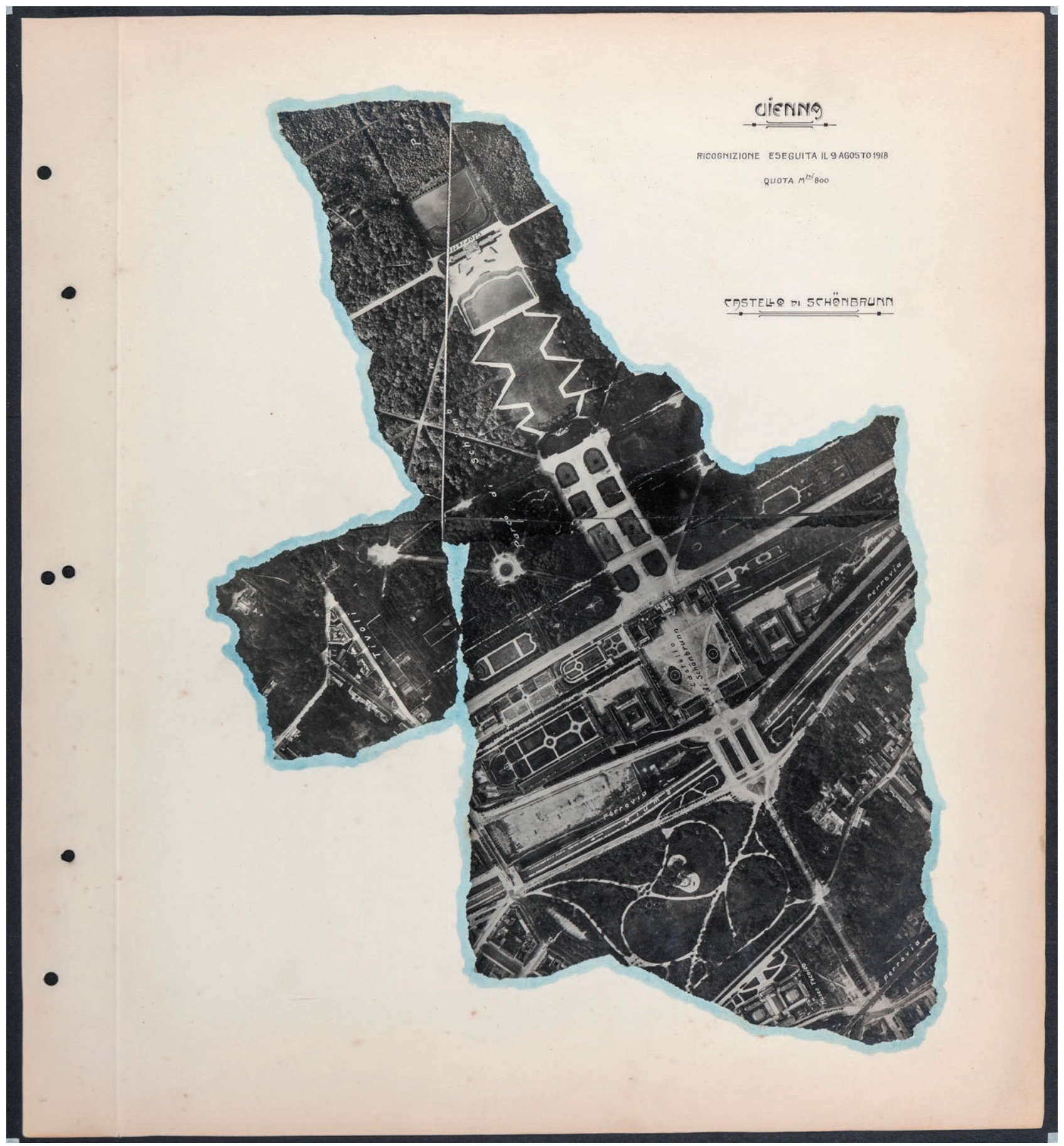

Figura 2. Foto zenitali del Castello di Schönbrunn, Vienna, 9 agosto 1918, autori Cap. Natale Palli e Gabriele D’Annunzio. Fonte: Archivio del Museo della $3^{\text {a }}$ Armata, Padova, Fondo cartografico, Foto aeree Cap. Palli, 41. 
il luogo dello scatto e il territorio rappresentato (il monte Hermada, attualmente definito Ermada). Sulla foto, in inchiostro rosso, sono indicati i nomi dei centri urbani (Trieste, Duino), della viabilità (ferrovia, viadotto) e delle posizioni nemiche, perlopiù al vertice dei rilievi (q. 323, q. 289). La foto è databile a prima di Caporetto, quando il monte Harmada era utilizzato come caposaldo naturale dall'esercito austroungarico. Questo tipo di documenti era utilizzato sia per permettere l'immediato riconoscimento del territorio ai comandi in prima linea, sia per trasmettere ai comandi nelle retrovie adeguati strumenti conoscitivi sulla situazione in proelio.

Esse erano anche utilizzate come modelli per elaborare degli "schizzi panoramici", ovvero dei disegni che combinavano le tecniche del tratteggio, dell'ombreggiatura e del lumeggiamento per riprodurre determinate porzioni del fronte. Anche in questo caso i punti notevoli erano evidenziati con la trascrizione del toponimo; nella fig. 3b, "Schizzo panoramico dalla quota 507 del Monte Sabotino" è presente anche l'orientamento, con una freccia che indica il meridione.

Elementi fondamentali per la comprensione di entrambi questi documenti sono l'orientamento, solo a volte esplicitato, il numero di foto che li compongono, la distanza e la posizione da cui sono state scattate, l'estensione dell'area riprodotta, la presenza o meno di indicazioni scritte e gli obiettivi della loro realizzazione, che potevano essere sia bellici sia propagandistici.

\subsection{Le cartografie tattiche}

Le fonti cartografiche che compongono il corpus possono essere classificate in alcune macrotipologie: carte tattiche e distributive, carte del genio, carte ad uso degli alleati e carte di spionaggio.

Le carte tattiche e distributive sono quelle disponibili in numero maggiore e che costituiscono il nucleo più eterogeneo. Con questa definizione si fa riferimento a quelle carte a stampa aggiornate quotidianamente a mano libera che restituiscono la situazione di particolari elementi tattici in proelio, che potevano riguardare la situazione delle truppe, dell'artiglieria e delle opere difensive.

Un esempio è rappresentato dalla carta riprodotta nella fig. 4. Sulla base di una tavoletta IGM al 25.000, è stata riportata in forma manoscritta la posizione di ogni

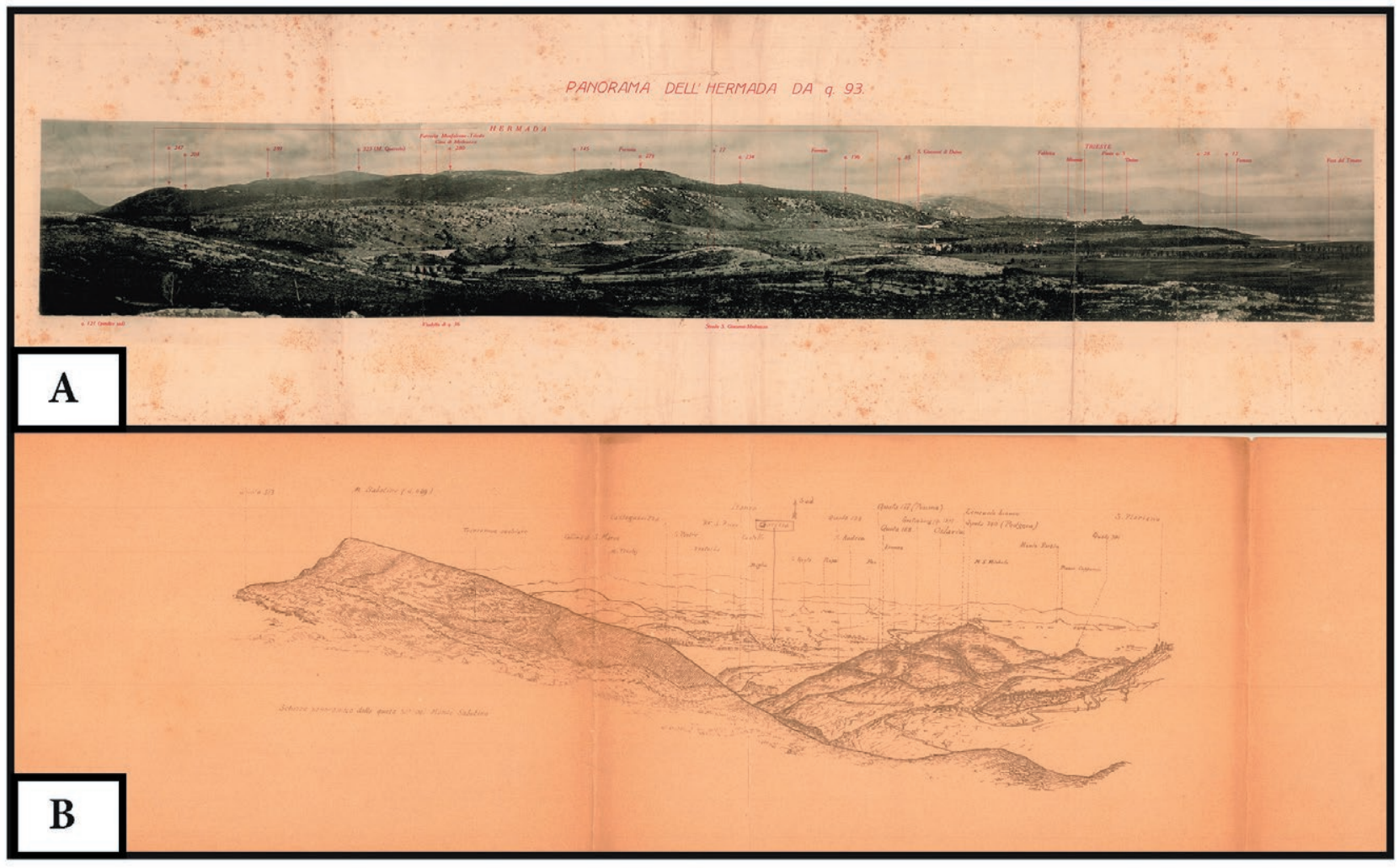

Figura 3. A: Panorama dell'Hermada da q. 93, foto panoramica, [giugno-settembre 1917?]. B: Schizzo panoramico della quota 507 del Monte Sabotino, [1916?], tratteggio e chiaroscuro a matita. Fonte: Archivio del Museo della $3^{\text {a }}$ Armata, Padova, Fondo cartografico, f. 25P e f. 5P. 
singolo battaglione della $3^{\text {a }}$ Armata attestato sul Piave. Un cartiglio, in basso a destra, indica la data e l'ora della rappresentazione, ovvero le ore 8 del 16 giugno 1918 . Il documento fa parte di una ampia serie, che a cadenza quotidiana raffigura lo schieramento dell'Armata dal maggio 1918 fino al dicembre 1919. L'areale di competenza della $3^{\text {a }}$ Armata è delimitato da linee in inchiostro nero, continue sul fronte, tratteggiate nelle retrovie; a sua volta è diviso in settori indicati con lettere $(Z, M$ e B) e in sotto-settori numerati. I diversi colori, tratteggiati a pastello, indicano le diverse brigate a cui i reggimenti afferiscono; il loro nome è trascritto a matita a fianco.

Durante la guerra, uno degli utilizzi più significativi della cartografia fu relativo al direzionamento delle artiglierie: sulla base dei rilievi altimetrici e della riproduzione della topografia del terreno, gli artiglieri dovevano calcolare i sistemi di puntamento e le gittate per i tiri indiretti contro le linee nemiche (Reali, Ricci 2018). I bombardamenti erano utilizzati per indebolire trincee e barriere in modo da permettere l'avanzata delle fanterie; questo rendeva necessario poter calcolare con precisione il bersaglio, per evitare vittime di fuoco amico e colpire gli obiettivi tattici più rilevanti, evitando lo spreco di proiettili per i tiri di aggiustamento. Per questo motivo, esse riportavano sia la posizione dei reparti italiani sia di quelli austroungarici.

La fig. 5 mostra un esempio peculiare di cartografia ad uso dell'artiglieria. Come la precedente riporta in cal-

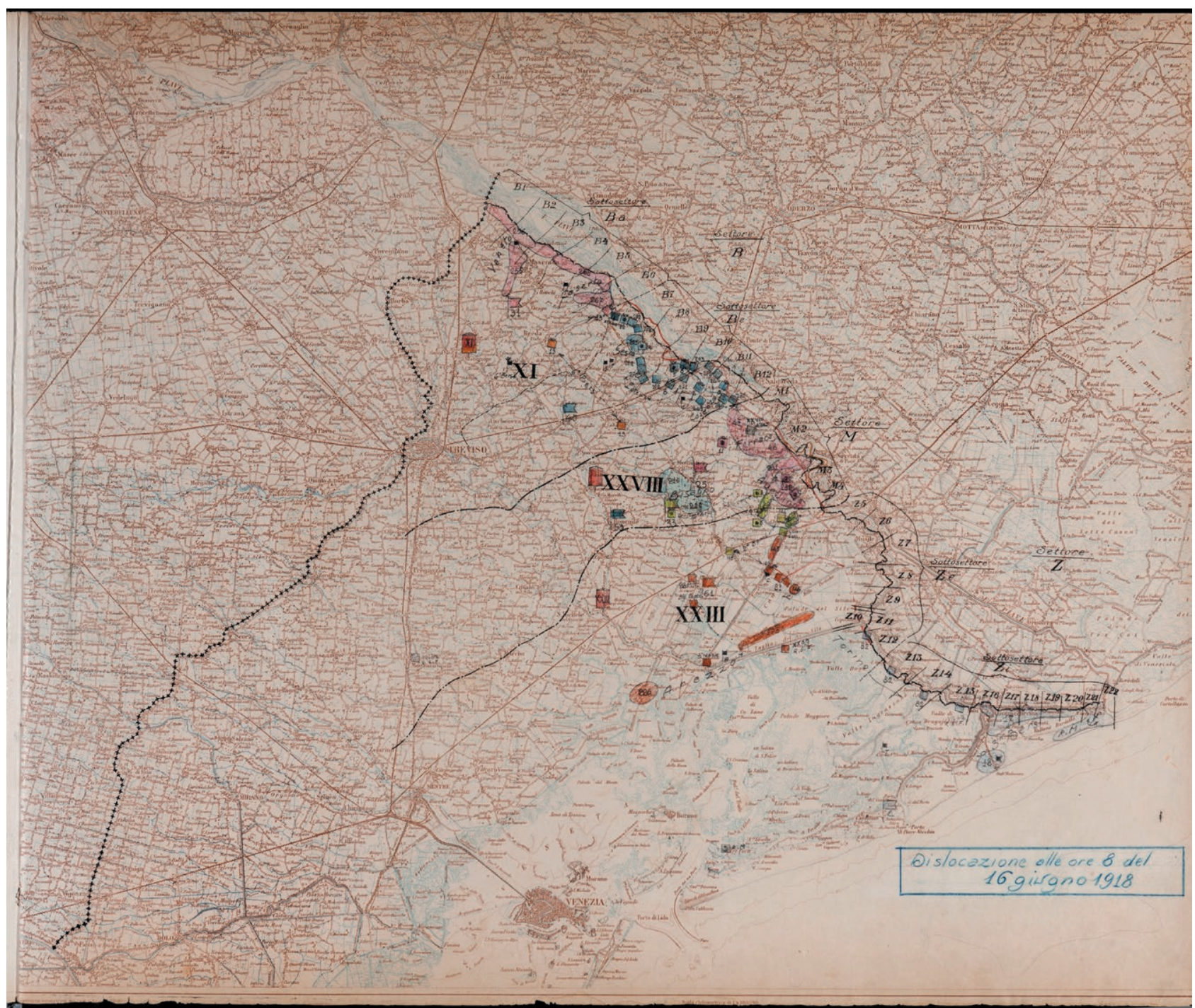

Figura 4. Dislocazione alle ore 8 del 16 giugno 1918, carta tattica di dislocazione delle truppe attestate sul Piave, 1:25.000. Fonte: Archivio del Museo della $3^{\text {a }}$ Armata, Padova, Fondo cartografico, Cartine ultimo semestre. 


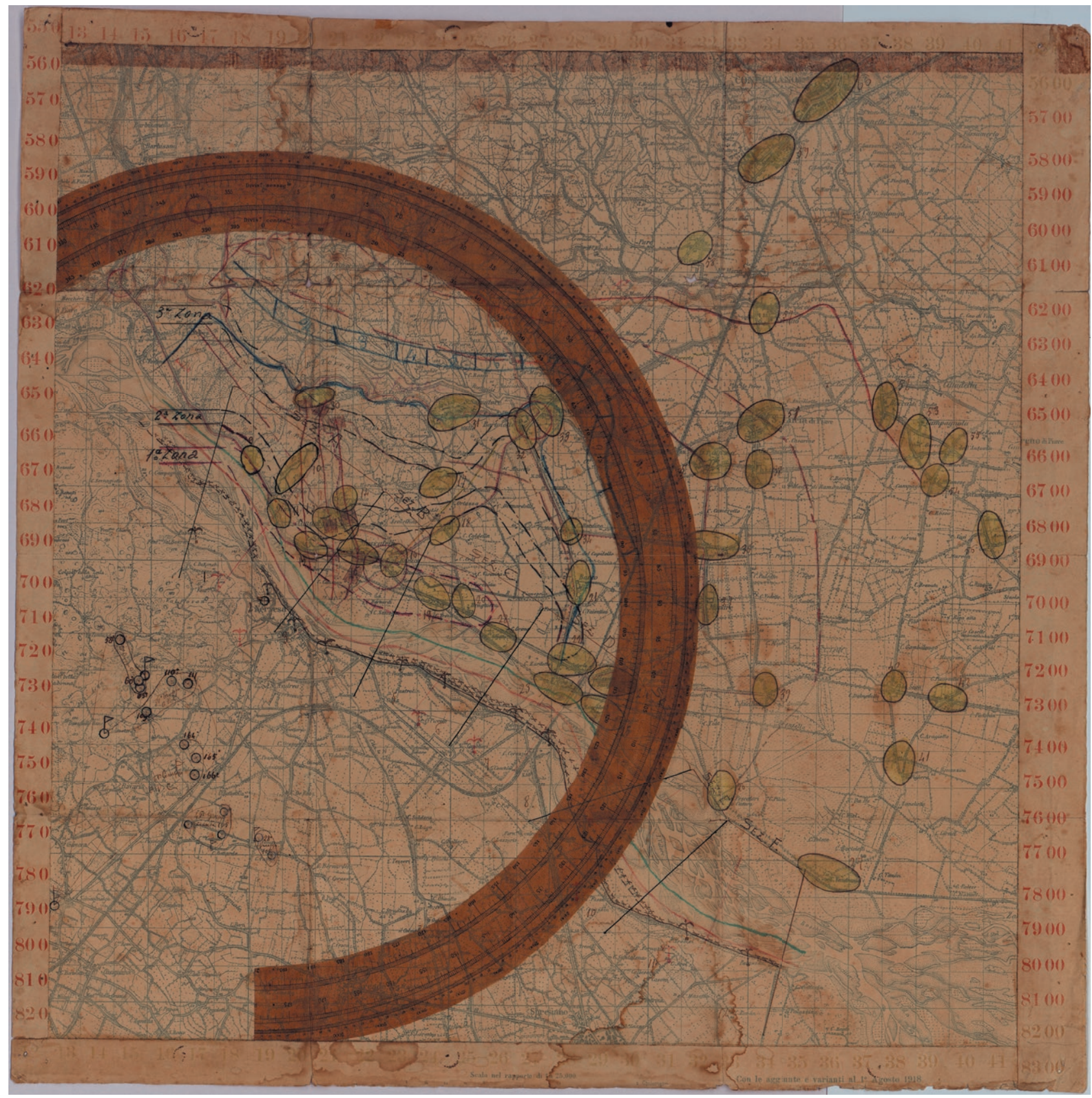

Figura 5. Carta tattica per il tiro di artiglieria, 1 agosto 1918, 1:25.000. Fonte: Archivio del Museo della $3^{\text {a }}$ Armata, Padova, Fondo cartografico, f. 55C.

ce la data, 1 agosto 1918; rispetto alle carte delle truppe è dotata di numerosi elementi aggiuntivi, dettati dalla estrema precisione richiesta per gli artiglieri, come la scala (1:25.000) e soprattutto la griglia geografica: questo elemento, introdotto per primo in Francia nel 1915 e adottato rapidamente dagli altri eserciti, permetteva di calcolare rapidamente posizione e distanze.
La carta riporta in inchiostro nero la posizione del fronte e delle artiglierie italiane (in cerchi colorati di azzurro, con accanto segnata la denominazione e le caratteristiche, come "Gruppo O.P.C.", Obici Pesanti Campali), e con poligoni colorati di giallo quella dei reparti austroungarici. Una serie di linee tracciate in rosso e blu pastello suddividono le retrovie nemiche in 


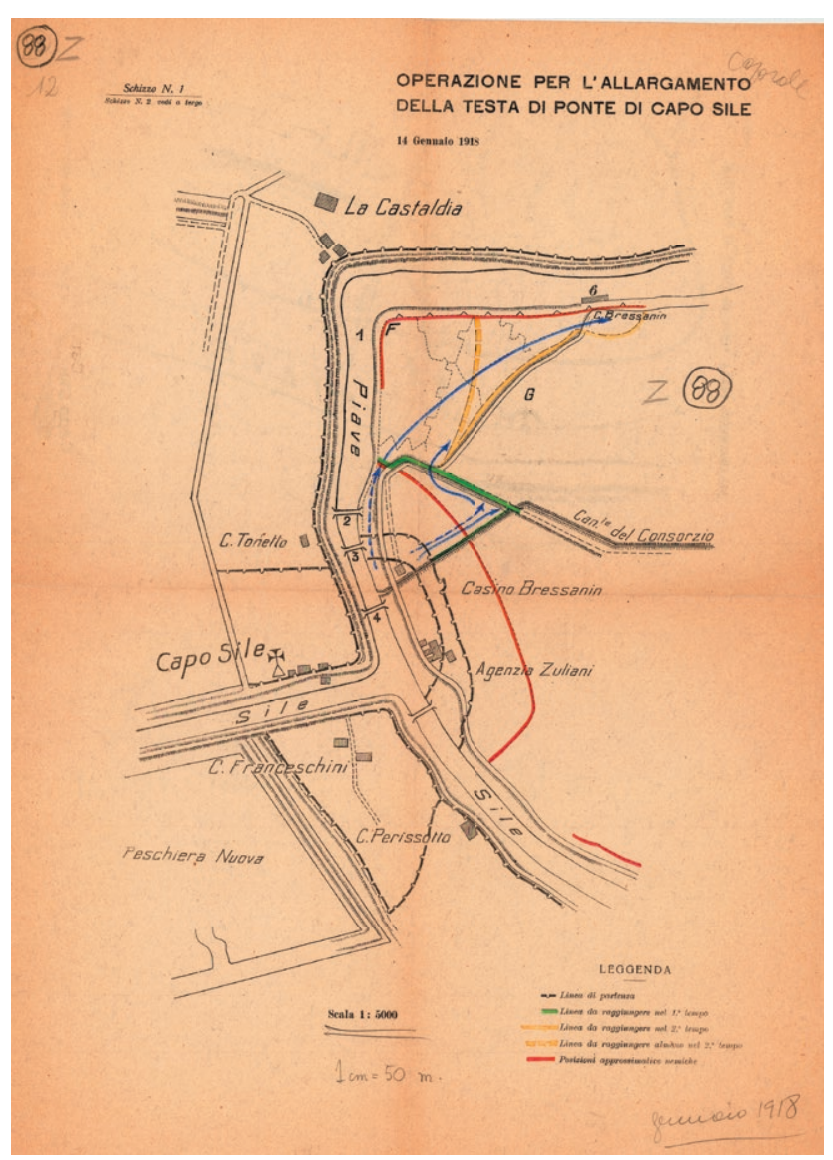

Figura 6. Operazione per l'allargamento della testa di ponte di Capo Sile, schizzo per carta tattica predittiva, 14 gennaio 1918, 1:5.000. Fonte: Archivio del Museo della $3^{\text {a }}$ Armata, Padova, Fondo cartografico, f. 39P.

varie zone, a seconda della distanza e della visibilità dai pezzi di artiglieria. Particolare è la presenza di un goniometro incollato sul supporto cartaceo, in modo da permettere un rapido calcolo dell'angolo di puntamento delle armi.

Le carte tattiche non riproducevano solo la situazione attuale: esse potevano anche essere "predittive", ovvero servire a illustrare le operazioni da compiere. Ne è un esempio lo schizzo "Operazioni per l'allargamento della testa di ponte di Capo Sile", datata al gennaio 1918 (fig. 6). La topografia a grandissima scala (1:5.000; in calce, a matita, è specificato che " $1 \mathrm{~cm}=50 \mathrm{~m}$ "), rappresenta l'area di Capo Sile, una delle poche enclaves mantenute dalle truppe italiane oltre il fiume Piave. Come riporta la legenda, le linee nere indicano i trinceramenti delle truppe italiane e quelle rosse le "posizioni approssimative nemiche". I segni verdi e gialli indicano rispettivamente la "Linea da raggiungere nel $1^{\circ}$ tempo" e la "Linea da raggiungere nel $2^{\circ}$ tempo", mostrando la pianificazione dell'attacco; frecce vergate in blu esplicitano il movimento delle truppe che, partendo dall'avamposto, devono cercare di addentrarsi in territorio nemico per almeno un chilometro.

Queste carte altamente dettagliate mostrano le posizioni austroungariche, compresi i trinceramenti, la posizione di artiglierie e mitragliatrici, i campi di aviazione, solitamente tracciati con inchiostro blu. In alcuni casi ogni trinceramento ha riportato a lato specifici toponimi legati al nome della brigata occupante, a personalità eminenti o a nomi di santi.

Oltre a quelle illustrate, sono presenti numerose topografie e corografie tattiche redatte per soddisfare le esigenze delle attività logistiche delle truppe, che riportano informazioni su trincee, viabilità, infrastrutture, riserve e magazzini.

Solitamente le carte mostrano una maggiore ricchezza di dettaglio per quanto riguarda le posizioni del nemico rispetto alle difese italiane. Questa scelta trovava la propria ragione nella volontà di evitare di fornire troppe informazioni all'esercito austro-ungarico nel caso in cui esse fossero cadute in mani nemiche. Le carte più dettagliate riportano la scritta "Riservato Personale. Riproduzione vietata" in calce; in tale caso, esse non dovevano essere portate in prima linea, per minimizzare i rischi di far trapelare informazioni sulle proprie forze o su localizzazioni potenzialmente fondamentali.

Una ulteriore peculiarità è data dalla ricca presenza di toponimi, sia manoscritti sia a stampa, spesso indicanti anche ogni singola casa isolata; durante le operazioni, essi potevano giocare un ruolo chiave nel localizzare e dare indicazioni a ogni singolo reparto. Inoltre, in qualche carta si trovano riportati dei toponimi "bellici" transitori, ovvero creati ex novo ispirandosi ai nomi delle unità italiane, come nel caso della fig. $7 \mathrm{a}$, che mostra le isole fluviali del Piave intitolate ognuna a una differente brigata dell'esercito, o a particolari caratteristiche ed eventi a scopo descrittivo o di avvertimento, come quelli illustrati nella fig. 7b, quali "Vallone della morte", "Massi rocciosi" e "Casa 5 finestre".

\subsection{Carte del genio}

Distinte dalle precedenti sono le piante prodotte da parte e per l'uso dei reparti del Genio, che riproducono la situazione della viabilità, delle infrastrutture e delle comunicazioni. Molte di esse risalgono al primo anno di guerra e sono state conservate durante tutto il conflitto dato il loro valore informativo strategico, meno transito- 


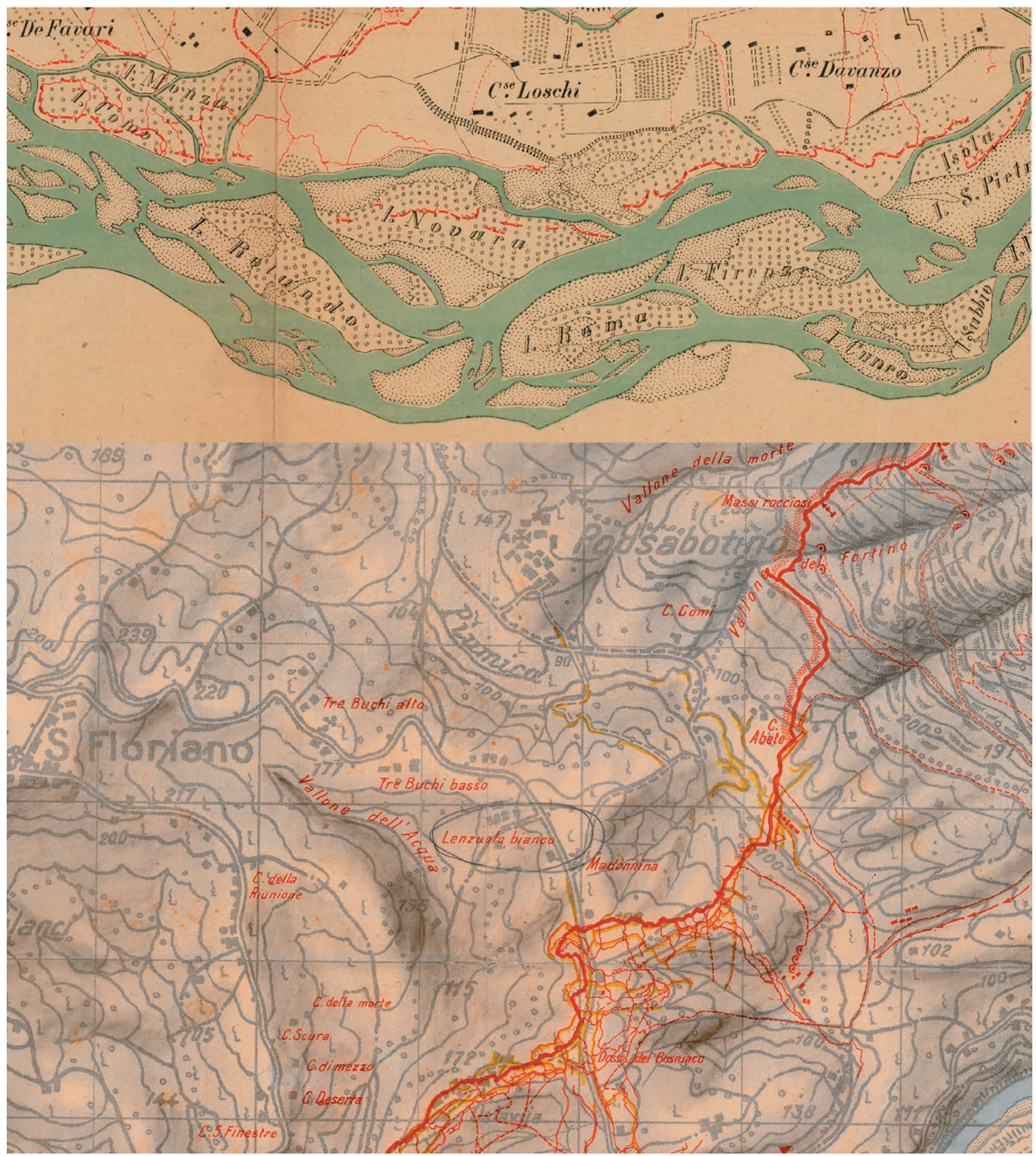

Figura 7. A: Ufficio Informazioni $3^{\text {a }}$ Armata, Schizzo del Piave con la vegetazione sulla sponda sinistra, s.d., 1:10.000, particolare dei toponimi delle isole fluviali; B: Schizzo dimostrativo della zona Vippacco-M. Sabotino, 1 maggio 1916, 1:10.000, particolare dei toponimi manoscritti. Fonte: Archivio del Museo della $3^{\mathrm{a}}$ Armata, Padova, Fondo cartografico, f. 42P e 105G.

rio rispetto a quelle tattiche. Inoltre, sono presenti anche planimetrie, a scala 1:500 o superiore, per la progettazione di sistemi difensivi, trincee o bunker.
La fig. 8 presenta un interessante schizzo che non corrisponde a una vera carta topografica, quanto piuttosto a uno schema di tutti i ponti che attraversano il fiu- 


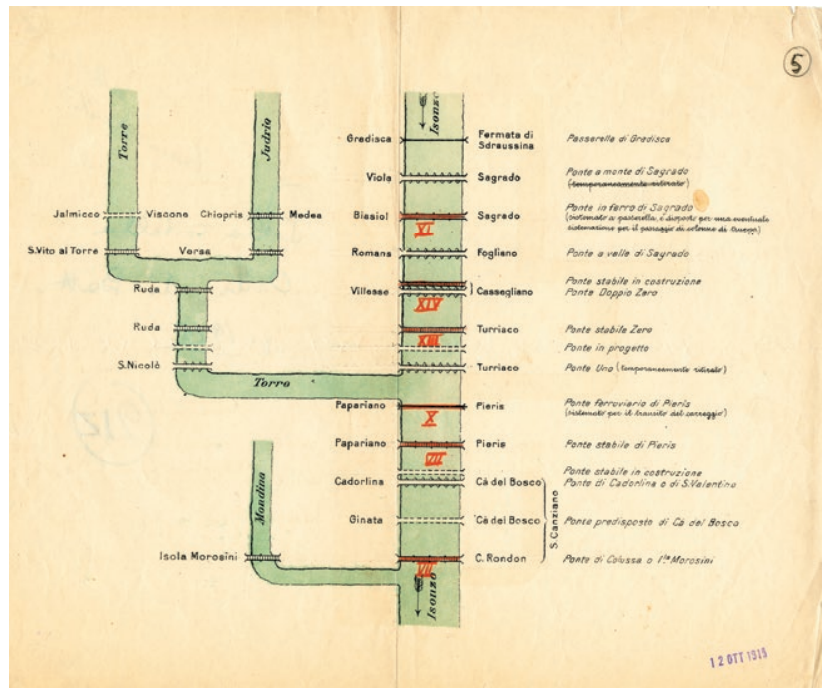

Figura 8. Schema esemplificativo delle posizioni e delle caratteristiche degli attraversamenti dell'Isonzo, 12 ottobre 1915. Fonte: Archivio del Museo della $3^{\text {a }}$ Armata, Padova, Fondo cartografico, f. 45P.

me Isonzo. La distanza tra di essi non riproduce misure reali; per ogni infrastruttura sono indicati i nomi dei due centri abitati più vicini sulle due sponde; a margine, una breve nota ne esplicita il nome e alcune caratteristiche, come "Ponte in ferro di Sagrado (sistemato a passerella, è disposto per una eventuale sistemazione per passaggio di colonne di truppa)". I ponti atti alle operazioni belliche sono colorati in rosso e per ognuno di questi la divisione di competenza è indicata con un numero romano.

In questa categoria, una ulteriore tipologia di carte è costituita da quelle riferite alla progettazione degli allagamenti pianificati, che sarebbero stati approntati come ultima difesa in caso di sfondamento della linea del Piave per rallentare l'avanzata austro-ungarica. In tal caso i reparti del Genio avrebbero dovuto rompere gli argini del Po e di corsi d'acqua minori in alcuni punti specifici, seguendo una strategia già messa fruttuosamente in atto dall'esercito sardo durante la Seconda Guerra di Indipendenza. Un esempio è rappresentato dalla carta in fig. 9, che mostra il settore delle retrovie immediatamente retrostante il fronte. Sulla tavoletta IGM al 25.000 il Piave è colorato in azzurro, così come l'area che sarebbe stata oggetto di allagamento, posta in basso a destra. I canali e i corsi d'acqua interessati dal sabotaggio sono evidenziati in diversi colori, a seconda del corpo del Genio che ne sarebbe stato responsabile. Le località più importanti lungo i loro tracciati hanno il toponimo evidenziato in inchiostro nero.

\subsection{Carte di spionaggio}

La Grande Guerra è stata combattuta, molto più delle precedenti, anche sulla base della capacità di (pre) vedere le mosse del nemico; un efficiente servizio informativo poteva consentire di monitorare il fronte e le retrovie avversarie, anticiparne le strategie e individuarne i punti di criticità da sfruttare. Il mezzo cartografico si prestava alla registrazione quotidiana dei movimenti delle truppe e dei sistemi difensivi, ma necessitava di un aggiornamento costante. La fonte principale per reperire informazioni era rappresentata dalle ricognizioni aeree; le aereofotogrammetrie prodotte erano poi sottoposte al lavoro degli ufficiali interpretatori, che ne isolavano gli elementi cospicui da riportare sulla base cartografica; questi dati erano poi incrociati con quelli raccolti durante gli interrogatori dei prigionieri, e con i rilevamenti in prima linea. Molte delle carte riportano in calce la scritta "Sistemazione difensiva austriaca desunta da fotografie di aviatori e informazioni di prigionieri" e la firma della Sezione $2^{\circ}$ (Informazioni) del Comando della $3^{\text {a }}$ Armata.

La fig. 10 presenta un interessante caso di carta di spionaggio raffigurante l'area del porto di Cortellazzo, situato alla foce del fiume Piave e quindi di estrema importanza strategica quale sito nevralgico del fronte difensivo italiano e possibile area di offensiva in caso di sfondamento delle linee nemiche. La base è rappresentata da un ingrandimento al 10.000 di tavoletta IGM; la legenda a stampa riporta i simboli utilizzati per le posizioni austriache (suddivise in "Trincea", "Trincea incerta", "Camminamento", "Appostamenti per tiratori", "Ricoveri e baraccamenti"), i punti di passaggio ("Passerella", "Ponte in legno") e evidenziata in verde, la "Zona paludosa (in gran parte intransitabile)". La data in calce è relativa al giorno 8 luglio 1918. Successivamente sono state effettuate numerose aggiunte e correzioni, come la data, cambiata nel 20 luglio. L'estensione dell'allagamento è stata modificata, segnando con matita rossa le aree dove "cancellare l'allagamento"; la voce della legenda "Zona paludosa" è modificata "in parte transitabile". Ulteriori trincee nemiche sono state aggiunte in rosso e poi in nero. Una scritta a matita sul bordo specifica la presenza di ulteriori 13 passerelle presso Revedoli, avvertendo che "si è dovuto adottare un segno identico tanto per le (tre?) passerelle quanto per i (due?) mascheramenti laterali". Questo documento costituisce quindi uno straordinario caso di fonte "palinsesto", che ci mostra sia il progressivo aggiornamento della copertura del suolo (e la contrazione dell'area inondata data dal procedere dell'estate) e dei trinceramenti, sia il metodo di 


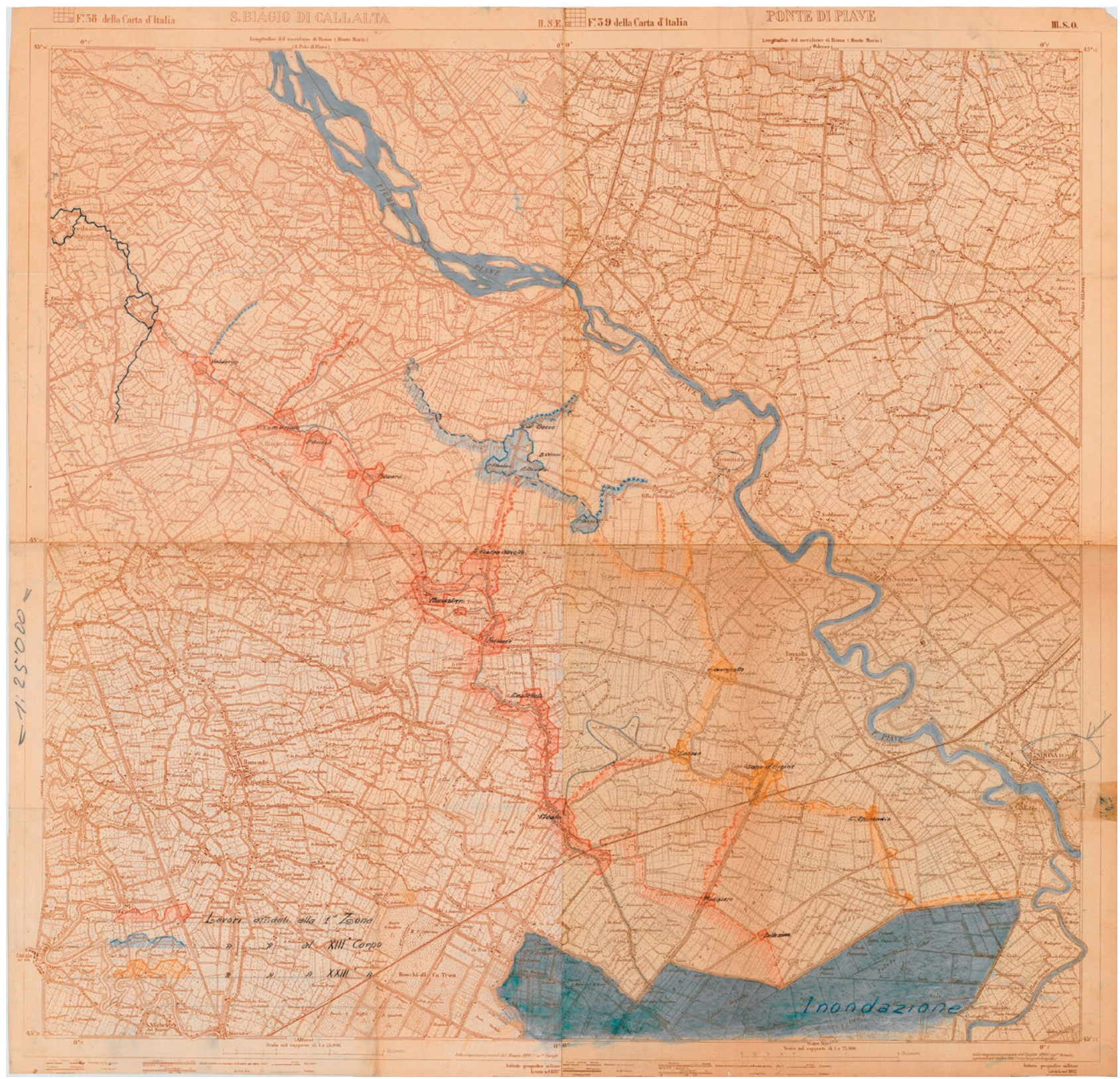

Figura 9. Carta di pianificazione degli allagamenti, su base IGM 1:25.000, s.d. Fonte: Archivio del Museo della $3^{\mathrm{a}}$ Armata, Padova, Fondo cartografico, f. 78G.

intervento degli ufficiali topografi (con vari colori a evidenziare le varie aggiunte) e le problematiche relative alla scelta dei simboli.

La stratificazione della carta è evidente anche nella fig. 11, che rappresenta la zona di Stari Lovka dove si svolse l'11 $1^{\text {a }}$ Battaglia dell'Isonzo. La base è rappresentata dall'adattamento di una carta austro-ungarica tricroma (la Spezialkarte der Österreichische-Ungarischen Monarchie) a scala 1:5.000 (da ingrandimento fotomeccanico di quella al 10.000 , e che presenta una ricchezza di dettaglio maggiore rispetto alle analoghe IGM, cfr. Guarducci 2018, 189). In particolare, in cerchi arancioni sono segnati gli acquartieramenti dei vari gruppi di soldati, ognuno contraddistinto da un nome specifico, legato ad un cognome (per esempio "Berger D.") o ad una funzione ("Brigade D. Telefon"). Su di essa, nell'agosto del 1917 sono state aggiunte a inchiostro rosso le linee raffiguranti le trincee e i reticolati e i simboli relativi 


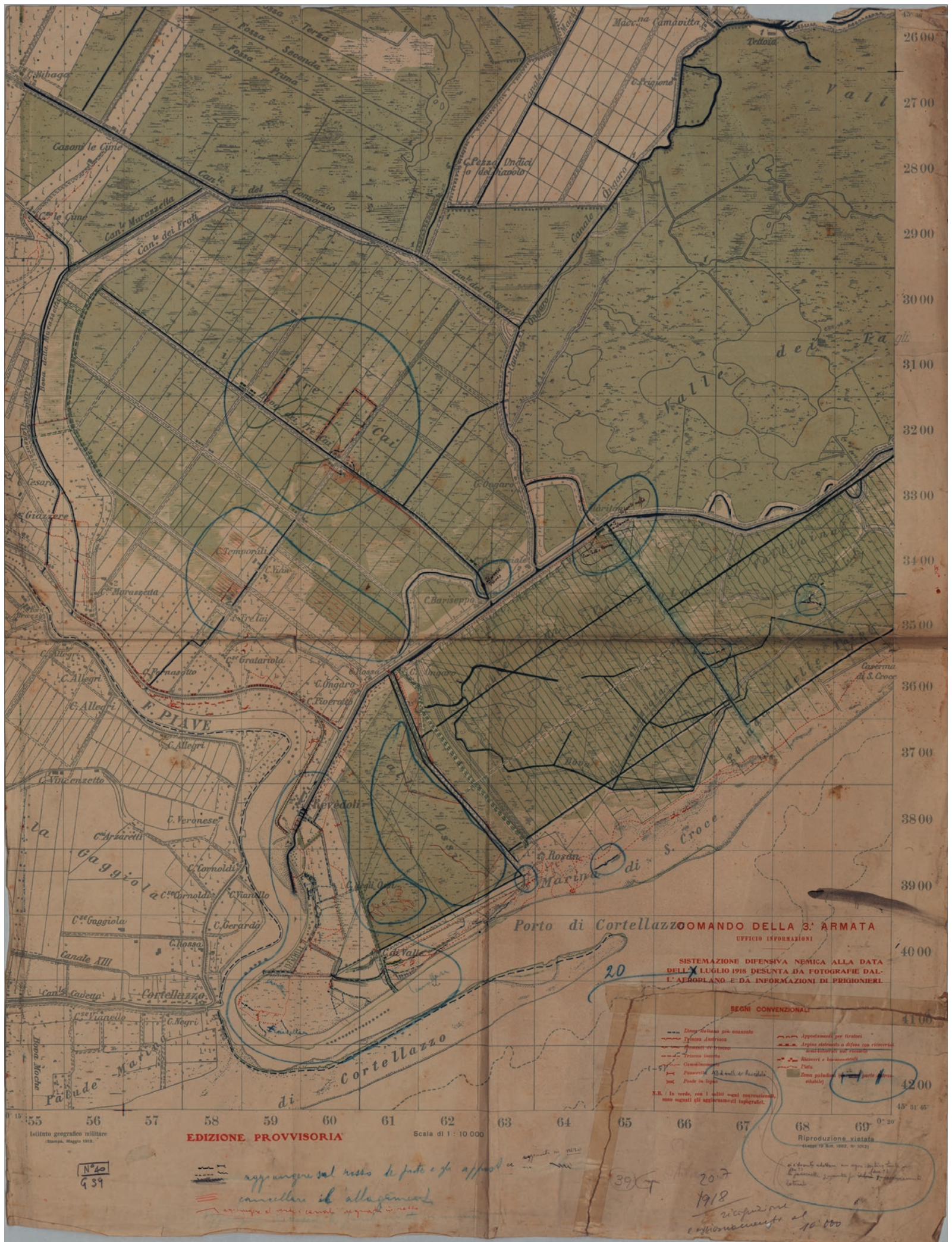

Figura 10. Ufficio Informazioni della $3^{a}$ Armata, Sistemazione difensiva nemica alla data dell'8 20 luglio 1918, 1:10.000. Fonte: Archivio del Museo della $3^{\text {a }}$ Armata, Padova, Fondo cartografico, f. 82G. 


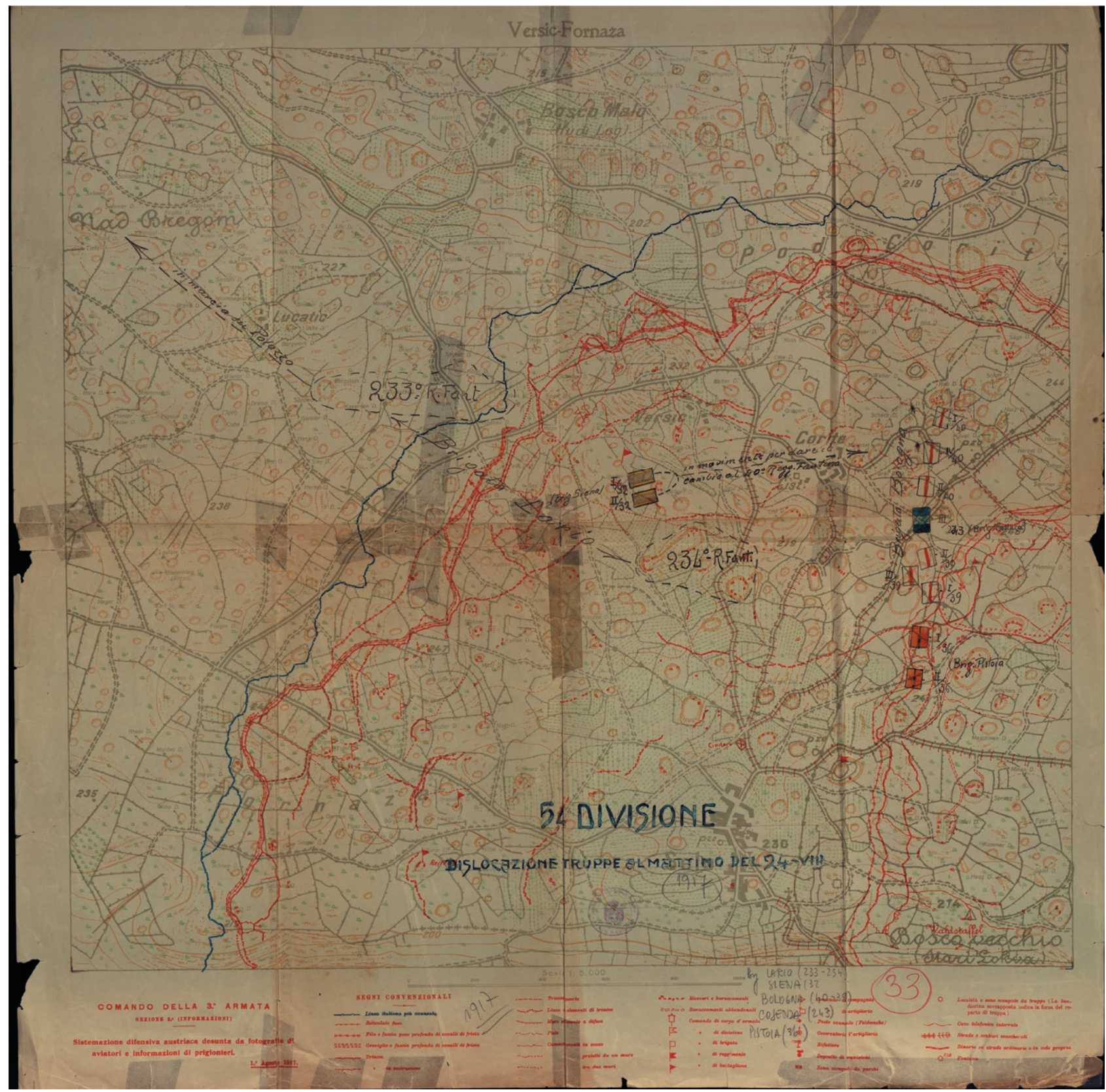

Figura 11. Carta palinsesto, con Ufficio Informazioni $3^{\mathrm{a}}$ Armata, Sistemazione difensiva austriaca, 1 agosto 1917, e Comando 54 Divisione, Dislocazione truppe al mattino del 24-VIII, 24 agosto 1917, 1:5.000. Fonte: Archivio del Museo della $3^{\text {a }}$ Armata, Padova, Fondo cartografico, f. 23 P.

ai comandi di compagnia, ai ricoveri e alle artiglierie desunte dalle foto aeree. Un terzo livello è costituito da informazioni aggiunte in inchiostro nero e pastelli colorati il 24 agosto 1917, ovvero la posizione della $5^{\text {a }}$ Divisione italiana dopo lo sfondamento delle linee asburgiche. Ogni reggimento, colorato diversamente a seconda della Brigata corrispondente, è riprodotto con un ret- tangolo; una serie di frecce tratteggiate ne indicano la direzione di movimento; a fianco si trovano indicati i rispettivi codici, insieme a informazioni di esplicazione come "I/32 e II/32 in movimento per dare il cambio al $40^{\circ}$ Regg. Fanteria". 
Tabella 1. Proposta di classificazione delle carte della Prima Guerra Mondiale sulla base dei fondi custoditi presso il Museo Storico della $3^{\text {a }}$ Armata di Padova.

\begin{tabular}{ccc}
\hline & Cartografie & Aerofotogrammetrie \\
\hline Macrotipologia & Tipologia & Zenitali \\
Panoramiche & Schizzi & Oblique \\
Carte tattiche e distributive & Di dislocazione delle truppe & \\
& Di dislocazione delle artiglierie & Di dislocazione delle opere difensive \\
& Modelli spaziali applicati all'artiglieria & Di spionaggio \\
& Di “lottizzazione" & Di ricognizione \\
Carte del genio & Predittive: di proiezione delle manovre & \\
& Ponti & Trasporti \\
Carte ad uso degli alleati & Telecomunicazioni & \\
Carte di spionaggio & Progetti di allagamenti difensivi & \\
& Copie di carte austriache &
\end{tabular}

\section{Per un'analisi semiologica: primi spunti sui simboli militari}

La letteratura internazionale è concorde nel ritenere che, generalmente, la simbologia delle carte militari precedenti alla Prima Guerra Mondiale non sia sofisticata come quella che è stato necessario sviluppare durante il conflitto (Collier 2015a). Proprio per questo motivo, la simbologia che si afferma in un tale momento di transizione si configura di particolare interesse per lo studio sia della pratica cartografica sia del conflitto (DelanoSmith 2007), rendendone potenzialmente fruttuosa l'identificazione e la decifrazione.

Lo sviluppo di nuovi armamenti e la specializzazione di vari reparti resero necessarie innovazioni nella tassonomia e nella simbologia cartografica, in modo da poter fornire quante più informazioni possibili ai comandanti nelle retrovie per meglio gestire tattiche e strategie in prima linea: i simboli cartografici militari, con dimensioni, cromatismi o forme capaci di mostrare la natura e l'entità delle varie unità militari, corredati da linee o frecce per mostrare i movimenti delle truppe, o le delimitazioni tra vari settori di competenza, fecero la loro comparsa nei primi mesi della Grande Guerra, riprodotti in forma manoscritta o a stampa su basi cartografiche statiche precedenti. Questa nuova pratica si sviluppò in itinere sul campo, per consolidarsi progressivamente nell'ultimo anno del conflitto (Hershey 2012). Alla fine delle ostilità, ogni esercito aveva ormai sviluppato il proprio inventario di simboli per descrivere graficamente il campo di battaglia; segni che iniziarono a standardizzarsi anche a livello internazionale, frutto degli scambi tra eserciti alleati, per cui alcuni accorgimenti si resero necessari in modo da rendere i propri supporti cartografici immediatamente comprensibili ai comandi cobelligeranti. È interessante notare, ad esempio, che le carte redatte dall'Esercito Italiano tra il 1915 e il 1917 prediligevano il colore blu o nero per le proprie truppe e il rosso per quelle nemiche; solo a partire dal 1918 si iniziò ad adottare il sistema cromatico inverso, cosiddetto "alla francese", similmente a quanto compì l'esercito britannico, in modo da conformare la simbologia utilizzata da tutti i paesi alleati per favorire la circolazione delle informazioni (Chasseaud 2018).

Gran parte delle carte illustrate nei paragrafi precedenti sono costituite da basi cartografiche a stampa a varia scala prodotte dall'IGM o dal K.u.K Militärgeographische Institut austroungarico; su di esse sono state aggiunte informazioni manoscritte, riguardanti sensibili cambiamenti dell'uso del suolo, il sistema difensivo (soprattutto trincee, bunker e posizioni di mitragliatrici), la viabilità, e le truppe operanti. Per quanto riguarda queste ultime, sulle topografie era spesso rappresentata la posizione di ogni singolo battaglione, inteso come unità minima di operazione sul terreno; i battaglioni sono indicati con numeri arabi e con un simbolo quadrato. Varie integrazioni consentivano di caratterizzare il più precisamente possibile la funzione dell'unità: il quadrato puntato rappresentava 
il battaglione con artiglieria, il quadrato con alamaro le truppe di fucilieri scelti e bersaglieri, l'ancora stilizzata inscritta nel quadrato le truppe di fanteria della Marina. I quadrati erano poi colorati con diversi cromatismi, a caratterizzare le brigate (indicate con il nome, che richiamava città o regioni) che raccoglievano i vari reggimenti.

Per differenziarli dalle unità di fanteria, i pezzi di artiglieria erano invece rappresentati con cerchi. Diversi colori permettevano di distinguere la natura del pezzo (cannone, bombarda, mortaio, etc.), il calibro e il materiale (acciaio, ghisa, bronzo, etc.). I simboli delle mitragliatrici erano cerchi intersecati con frecce, che ne evidenziavano anche la traiettoria di puntamento; elementi lineari continui erano utilizzati per riprodurre le trincee, mentre spezzati o puntati identificavano i diversi sentieri o le strade carrabili. Per le carte prodotte nel 1918, l'assenza della legenda a latere di ogni carta dimostra come la simbologia militare, seppure complessa, fosse ormai pienamente condivisa.

La localizzazione delle truppe italiane era identificata grazie ai rapporti dei comandi, oppure con voli interni di ricognizione; per le posizioni nemiche, oltre al rilevamento aereo, i dati erano raccolti anche attraverso carte originali austriache ottenute dall'Ufficio Informazioni con operazioni di intelligence o con la conquista di trincee nemiche (Guarducci 2018, 189-190), anch'esse presenti nel corpus dell'archivio. Queste venivano poi rielaborate per essere rese intellegibili ai comandi italiani, con riproduzioni essenziali oppure con vere e proprie copie che mantenevano la simbologia austro-ungarica e che riportavano a lato la legenda tradotta. Analizzando comparativamente le legende delle molte cartografie italiane e delle poche austro-ungariche pervenuteci, è possibile rilevare come ad una certa eterogeneità della simbologia in uso nel primo biennio (soprattutto per quanto riguarda $\mathrm{i}$ vari tipi di artiglieria e di opere difensive), si giunga poi a partire dal 1917-18 ad un consolidamento di alcuni simboli che vengono assunti nella prassi comune. Permangono, comunque, alcune differenze di tassonomia tra i due eserciti belligeranti, con un dettaglio maggiore delle carte austro-ungariche per le posizioni di mitragliatrici (indicate, perlopiù, da cerchi intersecanti frecce che indicano la direzione del tiro) che mostrano con tratti rettilinei anche l'area colpita dal fuoco (Espenhorst 2018).

Nella Figura 12 sono riprodotti alcuni dei segni cartografici utilizzati sulle carte militari dell'Esercito Italiano, per confrontare quelli illustrati su un volume di linee guida distribuito alle truppe dal Ministero della Guerra (1912) con quelli il cui uso appare ormai consolidato sulle carte tattiche del 1918. Alcuni di essi rimasero costanti (i segni riferiti al Comando di Corpo d'Armata e di Brigata) ed altri subirono solo minime modifiche

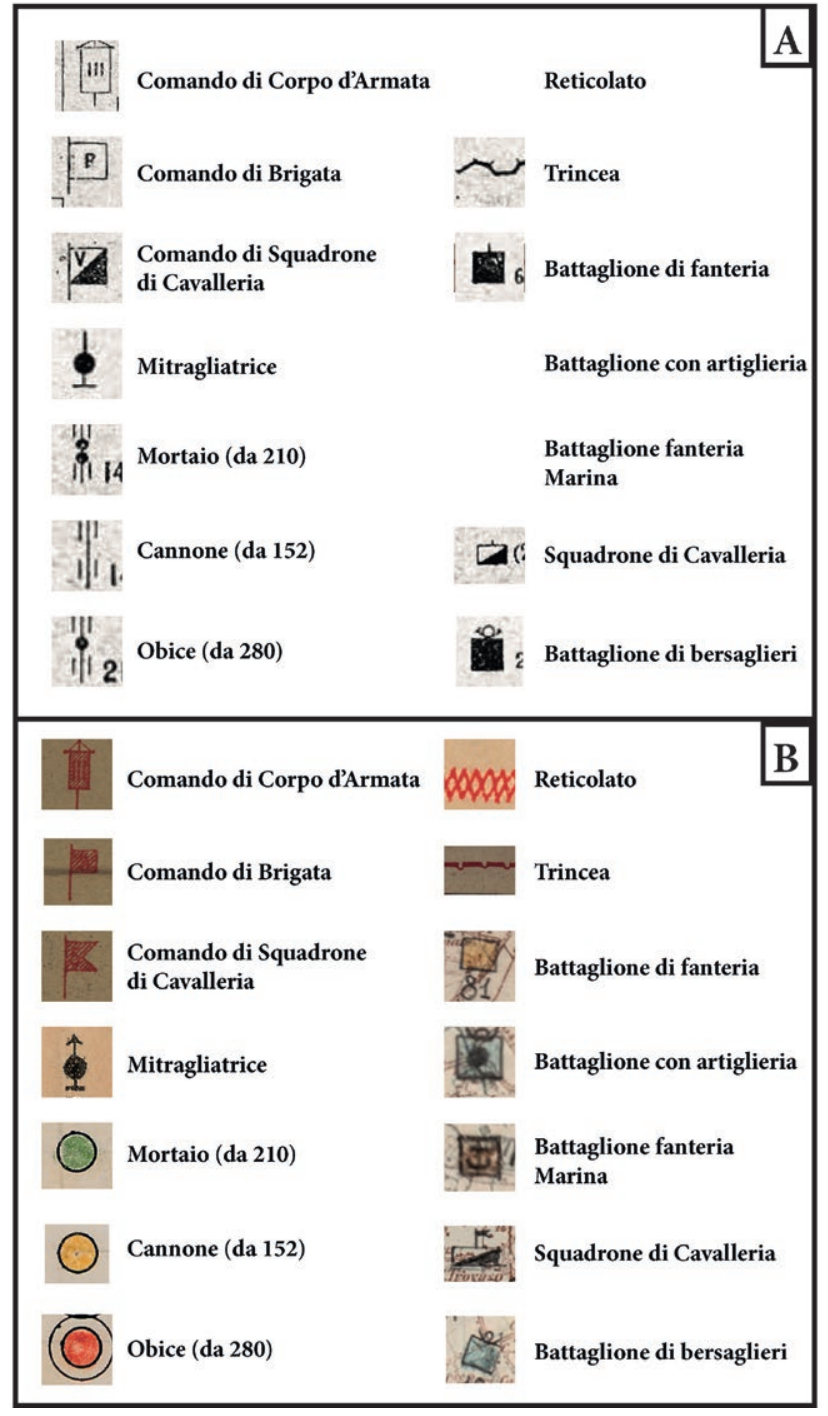

Figura 12. Esempi di simbologie cartografiche militari della Grande Guerra indicanti comandi, opere di difesa, artiglieria e truppe operanti; A: come esplicitati su un manuale del 1912; B: raccolti sulle carte militari e interpretati attraverso la legenda o una lettura comparata (1915-1919). Fonte: Elaborazione degli autori sulla base di (A) Ministero della Guerra 1912, pp, 215-219 e ( B ) Archivio del Museo della $3^{\circ}$ Armata, Padova, Fondo cartografico, c.c. v.v.

(posizioni di mitragliatrici, trincee, Squadrone di Cavalleria, Battaglione di Fanteria e Battaglione di Bersaglieri); alcuni elementi che non erano stati menzionati nella simbologia anteguerra entrarono invece nella prassi comune e necessitarono la creazione di segni dedicati (reticolati, battaglioni specializzati). Per quanto riguarda l'artiglieria, invece, la simbologia subì una forte alterazione, probabilmente a causa della complessità dei segni originari che furono soggetti a una forte semplificazione per poter essere leggibili sulle carte manoscritte. 
Secondo Chasseaud (2018), alla fine delle ostilità gli uffici cartografici militari dei tre paesi alleati (Regno Unito, Francia e Italia) avevano sviluppato una simbologia condivisa e standardizzata per gran parte degli elementi da rappresentare sulle carte. Questi simboli, diversi da quelli utilizzati da altri eserciti come quello tedesco, quello statunitense e quello sovietico, sono rimasti largamente inalterati fino alla Seconda Guerra Mondiale, quando le ulteriori innovazioni nelle pratiche belliche e nelle tecnologie si riflessero in nuove prassi cartografici (Hershey 2012). L'istituzione della North Atlantic Treaty Organization (NATO), nel 1949, rese obbligatoria la standardizzazione di una simbologia e di una tassonomia comune per tutte le forze armate dei paesi aderenti, che andarono sempre più adeguandosi a quella di ispirazione statunitense. Attualmente, lo standard cartografico internazionale è stato sancito dal protocollo APP-6A NATO Standard (War Department 1941; Traversi 1968; Manuale sulla simbologia terrestre 2000).

\section{Conclusioni e prospettive}

Le fonti geo-storiche iconografiche militari, quali cartografia e aerofotogrammetria, costituiscono un dispositivo euristico e conoscitivo dalle molteplici valenze, sia per quanto riguarda la riscoperta degli eventi bellici e della loro prospezione spaziale, sia per quanto attiene la comprensione delle dinamiche territoriali e paesaggistiche storiche in funzione di una applicazione nella gestione e pianificazione territoriale attuali.

L'immenso giacimento cartografico in custodia presso le varie articolazioni dell'Esercito Italiano, disperso in varie sedi e in gran parte inesplorato, si configura come un patrimonio culturale materiale e immateriale di fonti che meritano una congrua valorizzazione e visibilità, e il cui studio potenzialmente potrebbe aprire nuove frontiere di ricerca:

- per la ricostruzione delle tattiche e delle strategie belliche delle Forze Armate in tempo di guerra, e delle funzioni e competenze in tempo di pace (per esempio sulla localizzazione delle fortificazioni, sugli interventi sugli assetti territoriali pre- e post-bellici e di protezione civile, etc.) sia all'interno che all'esterno del territorio nazionale;

- per la storia delle tecniche e delle conoscenze cartografiche degli ufficiali e cartografi dell'Esercito Italiano e degli eserciti degli Stati pre-unitari;

- per la geo-storia dei paesaggi, degli insediamenti e delle infrastrutture dei territori rappresentati nelle cartografie (Francis 2014);
- per l'interpretazione tassonomica e semiologica dei linguaggi e delle simbologie cartografiche utilizzati in passato, e ancora in buona parte inesplorati.

La fase sperimentale delle ricerche ha permesso di prendere contezza di alcune tipologie di materiali relativi alla produzione cartografica della Prima Guerra Mondiale, per sviluppare alcune direttrici di ricerca inedite.

In particolare, appare urgente non solo salvaguardare dall'oblio e dalla dispersione i documenti cartografici, ma anche affinare con adeguato approccio critico strumenti e categorie di analisi e classificazione tassonomica per l'analisi semiologica e l'interpretazione critica di tale documentazione, al fine di permetterne una piena comprensione e un'agevole fruibilità per gli studiosi e gli stessi appartenenti alle Forze Armate interessati. Tale ambizioso percorso costituirebbe una preziosa ed originale occasione di reciproco beneficio e di scambio, condivisione e messa a sistema di competenze tra Esercito e Accademia, imprescindibile per approcciare e sviluppare adeguatamente un tema così complesso.

L'auspicio è che queste prime sperimentazioni costituiscano solo il primo passo di un percorso di ricerca di portata nazionale sul lungo periodo e di più ampia prospettiva, da sviluppare affinando un modello metodologico esportabile ed applicabile ai tanti materiali dispersi nei vari musei, biblioteche e strutture militari.

\section{Ringraziamenti}

Gli autori ringraziano la Direzione del Museo della $3^{a}$ Armata per l'autorizzazione alla pubblicazione delle immagini; Giovanni Santoro, dell'associazione Amici del Museo della $3^{\text {a }}$ Armata, il Colonnello Gaetano Celestre e il Maggiore Matteo Boschian Bailo del $2^{\circ}$ Reggimento Genio Guastatori Alpini per il supporto e l'aiuto; gli ignoti referee per le importanti sollecitazioni di riflessione e approfondimento fornite.

\section{Riferimenti bibliografici}

Allegri, D. (2018). Cartografare il fronte. L'evoluzione delle rilevazioni topografiche durante la Grande Guerra. In Corni, G. (a cura di). Preparare la guerra. Logistica e militarizzazione del territorio in Alta Valsugana. Trento, Curcu \& Genovese, 181-213.

Bergaglio, M. (2016). L'impiego dell'aereo nella rilevazione cartografica coloniale. La cartografia di guerra durante il conflitto italo-etiopico. Bollettino dell'Associazione Italiana di Cartografia, 111-113, 573-585. 
Bondesan, A., Scroccaro, M. (a cura di). (2016). Cartografia militare della I Guerra Mondiale. Cadore, Altopiani e Piave nelle carte topografiche austro-ungariche e italiane dell'Archivio di Stato di Firenze. Treviso, Edizioni Antiga.

Boria, E. (2012). Carte come armi. Geopolitica, cartografia, comunicazione. Roma, Edizioni Nuova Cultura.

Cantile, A. (2013). Lineamenti di storia della cartografia italiana. Roma, Geoweb, 2 voll.

Cantile, A. (2019). Il contributo dell'IGM alla Grande Guerra. In Dai Prà, E. (a cura di). Cesare Battisti, la Geografia e la Grande Guerra. Roma, CISGE, 35-46.

Carbone, L., Ciaschi, A. (2018). La Grande Guerra vista dall'alto. In Masetti, C. (a cura di). Per un Atlante della Grande Guerra. Roma, Labgeo Caraci, 167-174.

Chasseaud, P. (2013). Mapping the First World War: The Great War Through Maps From 1914 to 1918. Londra, Collins.

Chasseaud, P. (2018). British military mapping on the Western Front 1914-18. International Journal of Cartography, 4 (3), 245-271.

Carucci, P. (2013). Un nuovo studio per la disciplina archivistica e per la storiografia. In Trani, S. Il Regio Esercito e i suoi archivi. Una storia di tutela e salvaguardia della memoria contemporanea. Roma, Ministero della Difesa, Ufficio storico dello Stato Maggiore, 13-16.

Chirico, M.L., Conti, S. (a cura di). (2018). La Grande Guerra. Luoghi, eventi, testimonianze, voci. Roma, Aracne.

Collier, P. (2015a). Warfare and Cartography. In Monmonier, M. (a cura di). Cartography in the Twentieth Century. History of Cartography, vol. VI. Chicago e Londra, The University of Chicago Press, 1696-1700.

Collier, P. (2015b) World War I. In Monmonier, M. (a cura di). Cartography in the Twentieth Century. History of Cartography, vol. VI. Chicago e Londra, The University of Chicago Press, 1770-1775.

Collier, P. (2018). The development of photogrammetry in World War 1. International Journal of Cartography, 4 (3), 285-295.

Dai Prà, E. (a cura di). (2018). Cesare Battisti geografo e cartografo di frontiera. Roma, CISGE.

Dai Prà, E., Gabellieri, N. (2020). Cartografia, aerofotogrammetria e intelligence dell'Esercito Italiano durante la Grande Guerra. Gnosis. Rivista italiana di intelligence, 26 (1), 70-81.
Delano-Smith, C. (2007). Signs on Printed Topographical Maps, ca. 1470 - ca. 1640. In Woodward, D. (a cura di). Cartography in the European Renaissance. Vol. III, Part. I, The History of Cartography. Chicago e Londra, The University of Chicago Press, 258-280.

Demhardt, I.J. (2018). A terrible mother of invention: cartographic progress during World War I. International Journal of Cartography, 4 (3), 241-244.

Espenhorst, J. (2016). The Eye of the Army. German Aircraft and the Aero Cartography in World War I. In Liebenberg, E., Demhardt, I.J., Vervust, S. (a cura di). History of Military Cartography. Heidelberg, Springer International, 61-82.

Espenhorst, J. (2018). Germany and Austro-Hungary: the cartography of the defeated. International Journal of Cartography, 4 (3), 308-335.

Ferrandi, M. (1986). Ettore Tolomei l'uomo che inventò l'Alto Adige. Trento, Publilux.

Fontana, N., Pisetti, A. (a cura di). (2019). Archivi militari tra Ottocento e Novecento. Ricognizioni e acquisizioni. Trento, Soprintendenza per i Beni Culturali, Museo Storico Italiano della Guerra.

Fox, A. (2018). Learning to Fight. Military Innovation and Change in the British Army, 1914-1918. Cambridge, Cambridge University Press.

Francis, R.A. (2014). On war (and geography): engaging with an environmental frontier. Progress in Physical Geography, 38 (3), 265-270.

Gabellieri, N. (2019). Un "anello" della Galassia Battisti: Giovanni Battista Trener geologo e geografo. In Dai Prà, E. (a cura di). Cesare Battisti, la Geografia e la Grande Guerra. Roma, CISGE, 143-157.

Gentilli, R., Varriale, P. (1999). I reparti dell'Aviazione italiana nella Grande Guerra. Roma, Ufficio Storico dell'Aeronautica Militare.

Gibelli, A. (2007). La grande guerra degli italiani. Milano, Bur.

Guarducci, A. (2018). La cartografia della Grande Guerra nell'Archivio di Stato, nella Biblioteca di Geografia dell'Università e nella Biblioteca Nazionale Centrale di Firenze. In Masetti, C. (a cura di). Per un Atlante della Grande Guerra. Roma, Laboratorio geocartografico "Giuseppe Caraci", 189-199.

Heffernan, M. (1996). Geography, Cartography and Military Intelligence: The Royal Geographical Society and the First World War. Transactions of the Institute of British Geographers, 21 (3), 504-533. 
Hershey, A. (2012). Not Just Lines on a Map: A History of Military Mapping. Strategy \& Tactics, 274 (23), 22-27.

Jung, P. (2000). L'ultima guerra degli Asburgo. Basso Isonzo, Carso, Trieste. 1915-1918. Gorizia, LEG.

Labanca, N. (2014). Storia militare e fonti archivistiche: una relazione stretta, base di unalleanza fra storici militari e archivisti. Rassegna degli Archivi di Stato, nuova serie, 10 (1-2-3), 97-109.

Lacoste, Y. (1976). La géographie ça sert d'abord à faire la guerre. Parigi, Maspero.

Laureti, L. (2018). Confini naturali e politici del nostro paese: la posizione dei geografi italiani agli inizi del XX secolo. In Dai Prà, E. (a cura di). Cesare Battisti geografo e cartografo di frontiera. Roma, CISGE, 207-224.

Liebenberg, E., Demhardt, I.J., Vervust, S. (a cura di). (2016). History of Military Cartography. Heidelberg, Springer International.

Maddrell, A. (2008). The "Map Girls". British women geographers' war work, shifting gender boundaries and reflections on the history of geography. Transactions of the Institute of British Geographers, 33, 127-148.

Manuale sulla simbologia terrestre (2000). Roma, Stato Maggiore dell'Esercito Italiano.

Masetti, C. (a cura di). (2018). Per un Atlante della Grande Guerra. Roma, Laboratorio geocartografico "Giuseppe Caraci".

Ministero della Guerra, Comando del Corpo di Stato Maggiore (1912). Servizio in guerra. Vol I. Servizio delle truppe. Roma, Voghera Enrico Tipografo.

Proto, M. (2014). I confini d'Italia. Geografie della nazione dall'Unità alla Grande Guerra. Bologna, Bononia University Press.

Reali, R., Ricci, A. (2018). Cartografi e matematici. I nuovi utilizzi dell'artiglieria durante il primo conflitto mondiale. In Masetti, C. (a cura di). Per un Atlante della Grande Guerra. Roma, Laboratorio geocartografico "Giuseppe Caraci”, 189-199.

Rombai, L. (2016). Cesare Battisti (1875-1916). Geografo innovatore. Firenze, Università degli Studi di Firenze, Laboratorio di Geografia applicata-Phasar.

Schneider, U. (2017). Maps, Geography and the Representation of Space. In Daniel, U., Gatrell, P., Janz, O., Jones, H., Keene, J., Kramer, A., Nasson, B. (a cura di). 1914-1918-online. International Encyclopedia of the First World War. Berlino, Freie Universität Berlin, s.p.
The Map Sense. The Times. 6 aprile 1915, 9.

Thibaud, D.U. (2005). Commented APP-6A - Military symbols for land based systems. NATO's current military symbology standard. Valcartier, Defence R\&D Canada.

Trani, S. (2006). Gli archivi degli uffici storici e dei musei delle Forze Armate: appunti per una discussione. Le carte e la storia, 12, 40-47.

Trani, S. (2013). Il Regio Esercito e i suoi archivi. Una storia di tutela e salvaguardia della memoria contemporanea. Roma, Ministero della Difesa, Ufficio storico dello Stato Maggiore.

Traversi, C. (1968). Tecnica cartografica. Firenze, IGM.

War Department (1941). Basic field manual. Conventional signs, military symbols, and abbreviations. Washington, United States Government Printing Office. 